\title{
The determination of the large-scale circulation of the Pacific Ocean from satellite altimetry using model Green's functions
}

\author{
Detlef Stammer and Carl Wunsch \\ Center for Meteorology and Physical Oceanography, Department of Earth, Atmospheric and \\ Planetary Sciences, Massachusetts Institute of Technology, Cambridge
}

\begin{abstract}
A Green's function method for obtaining an estimate of the ocean circulation using both a general circulation model and altimetric data is demonstrated. The fundamental assumption is that the model is so accurate that the differences between the observations and the model-estimated fields obey a linear dynamics. In the present case, the calculations are demonstrated for model/data differences occurring on very a large scale, where the linearization hypothesis appears to be a good one. A semi-automatic linearization of the Bryan/Cox general circulation model is effected by calculating the model response to a series of isolated (in both space and time) geostrophically balanced vortices. These resulting impulse responses or "Green's functions" then provide the kernels for a linear inverse problem. The method is first demonstrated with a set of "twin experiments" and then with real data spanning the entire model domain and a year of TOPEX/POSEIDON observations. Our present focus is on the estimate of the time-mean and annual cycle of the model. Residuals of the inversion/assimilation are largest in the western tropical Pacific, and are believed to reflect primarily geoid error. Vertical resolution diminishes with depth with 1 year of data. The model mean is modified such that the subtropical gyre is weakened by about $1 \mathrm{~cm} / \mathrm{s}$ and the center of the gyre shifted southward by about $10^{\circ}$. Corrections to the flow field at the annual cycle suggest that the dynamical response is weak except in the tropics, where the estimated seasonal cycle of the low-latitude current system is of the order of $2 \mathrm{~cm} / \mathrm{s}$. The underestimation of observed fluctuations can be related to the inversion on the coarse spatial grid, which does not permit full resolution of the tropical physics. The methodology is easily extended to higher resolution, to use of spatially correlated errors, and to other data types.
\end{abstract}

\section{Introduction}

The TOPEX/POSEIDON (T/P) altimetric satellite is providing oceanographers for the first time with a continuing global database for describing and understanding the large-scale ocean circulation. The satellite was launched in August 1992 and ever since has been measuring the global sea surface height every 10 days with an unprecedented accuracy using a radar altimeter system along repeating ground tracks (see the Journal of Geophysical Research special issues on $\mathrm{T} / \mathrm{P}$, volume 99 , number $\mathrm{C} 12$ 1994, and volume 100 , number $\mathrm{C} 12$, 1995). With this data set, and with other global-scale in situ data from programs such as the World Ocean Circulation Experiment, one finally can begin describing the ocean circulation quantitatively on a nearly day-today basis, rather than as a vague climatological mean.

Copyright 1996 by the American Geophysical Union.

Paper number $96 \mathrm{JC} 01150$.

0148-0227/96/96JC-01150\$09.00
There is another major reservoir of knowledge of the ocean circulation: the general circulation models, which attempt to summarize understanding of ocean physics in the form of numerical codes. The problem we address here is to find a practical means for combining the information from diverse observations with the dynamics inherent in ocean general circulation models (OGCMs) so as to produce best estimates of the oceanic state at any given time and place accounting properly for errors in both models and observations. This problem is a challenging one because model and observational errors contain complex space/time structures and the number of variables required to describe the oceanic state at any one time (the "state vector") is enormous. The general problem we are addressing is one of estimation theory (meteorologists would call it "assimilation").

Algebraically, an OGCM can be written in canonical form as

$$
\mathbf{x}(t+\Delta t)=\Gamma(\mathbf{x}(t), \mathbf{q}(t), t)
$$

where $\mathbf{x}(t)$ is the state vector at discrete time $t, \Gamma$ represents the operator stepping the model forward in time starting from a prescribed initial condition $\mathbf{x}\left(t_{0}\right)$, and 
$\mathbf{q}(t)$ represents externally specified boundary conditions and sources and sinks.

Most oceanographic measurements, including altimetric ones, are at least approximately a linear combination of the model state vector, e.g., velocity, temperature, and salinity, but are contaminated by noise. Such measurements can be written as

$$
\mathbf{y}(t)=\mathbf{E}(t) \mathbf{x}(t)+\mathbf{n}(t),
$$

where $\mathbf{E}$ is the "observation matrix" relating the model state vector to observables. Normally, $\mathbf{E}$ is very sparse because observables usually involve only local subsets or related elements of $\mathbf{x}(t)$. Altimetric surface height observations $\zeta$, in particular, are obtained only at the sea surface, measuring the dynamically induced surface elevation (or the equivalent surface pressure $p_{s}=g \rho \zeta$ ) along subsatellite tracks sequentially in time, while floats or tomographic experiments provide subsurface data along float trajectories or tomographic ray paths. The precise structure of $\mathbf{E}$ and the existence of its inverse is a very important question which, in the context of altimetry, is directly related to the general issue of the observability of the ocean circulation through sea surface height measurements [Fukumori et al., 1993]. In the terminology of inverse problems, observability is equivalent to the state estimation problem having full rank.

The problem we wish to solve is to find an estimate $\hat{\mathbf{x}}(t)$ of the state vector of the ocean subject to model dynamics, and its uncertainty $\mathbf{P}(t)$, given observations $\mathbf{y}(t)$, with noise covariance $\mathbf{R}(t)$, and a model (1) with uncertainty $\mathbf{Q}(t)$. Most estimation methods addressing this problem require the minimization of a quadratic function measuring the model minus data misfit,

$$
\breve{J}=\sum_{t}(\mathbf{y}(t)-\mathbf{E} \hat{\mathbf{x}}(t))^{T} \mathbf{R}(t)^{-1}(\mathbf{y}(t)-\mathbf{E} \hat{\mathbf{x}}(t))
$$

subject to the model physics constraints. (Here $\hat{\mathbf{x}}(t)$ represents the estimate of the state vector that results from combining the model and observations.) There are many schemes for minimizing $J$ subject to the model in (1), through either constrained or unconstrained optimization methods. Optimization schemes known to be useful, however, overwhelm present-day computer facilities for realistic problems on basin scales and larger. For this reason, many suboptimal approaches are being studied, including state reduction, the use of steadystate sequential algorithms, and the like [e.g., Fukumori et al., 1993; Marotzke and Wunsch, 1993].

In addition to the problem of state vector dimension, the oceanographic case is complicated by the nonlinearities in (1). To deal with both the size of the problem and the nonlinearity, we use here a method which is based upon a form of Green's functions for a linearized model associated with the OGCM. The fundamental assumption is that modern nonlinear OGCMs driven by observed meteorological fields will be sufficiently accurate in simulating the ocean that the discrepancies between the computed and observed oceanic state obey linear physics. Almost all optimization methods which are practical with large systems (filters, smoothers, Pontryagin principle/adjoints) are based upon linearization of the model dynamics about a reference state. Finding the linearization can be an onerous job; one of our goals here is to render this job less demanding of programmer skill than it normally is.

As discussed in more detail below, the OGCM is used twice: once to compute, using the best available initial and boundary conditions, a model first estimate of the oceanic state. In a separate calculation, the model first estimate is then empirically linearized by perturbing it with a succession of simple disturbances on a coarser grid. This new, coarse resolution, linear model is used to represent the differences between the large-scale data and the original full OGCM during the estimation procedure. The result is a linear inverse problem for a set of disturbance coefficients, which is then solved. The original full nonlinear model can be iteratively improved if necessary. This linearization procedure, if it can be shown to be effective, would serve several practical purposes, including separating the problem of making model improvements in the OGCM from those of estimation, and using the numerical power of a computer to produce the model linearization, which is otherwise a major chore.

Note that this method is related to one used previously by Wunsch [1988] and Memery and Wunsch [1990] for estimating boundary conditions using transient tracers. Those authors determined the tracer boundary conditions which best fit observed tracer distributions (in space and time) in the interior ocean. However, as will become clear later, the tracer approach is complementary to ours: we aim to estimate the interior ocean state which best fits observed, but noisy, boundary conditions.

There is no guarantee that the linearization is valid: the OGCM/data differences must be assessed in detail. Furthermore, to usefully combine observations with a model, one must be able to show that their differences are consistent within the uncertainty estimates for both. A full model/data consistency check is an important study in its own right and will be presented elsewhere [e.g., Stammer et al., 1996].

Here we seek only to make it plausible that the deviations of data and model satisfy a linear set of equations. Stammer and Wunsch [1994] show the differences between a 3-year mean sea surface height field obtained from the global Semtner and Chervin [1992] model with $1 / 4^{\circ}$ horizontal resolution relative to a 1 -year mean of $\mathrm{T} / \mathrm{P}$ sea surface height observations. Deviations are generally found in the $10 \mathrm{~cm}$ range on large spatial scales with length and velocity scales of the order of $500 \mathrm{~km}$ and $10 \mathrm{~cm} / \mathrm{s}$, respectively, leading to a Rossby number, $R=U / f L$, of the order of $10^{-2}$ to $10^{-3}$ in midlatitudes. The small Rossby number limit is by itself insufficient to justify a linear physics on large scales; a referee has, in particular, noted that geostrophic motion of the second kind, i.e., obeying the classical thermocline equations, is intrinsically nonlinear. However, over the 

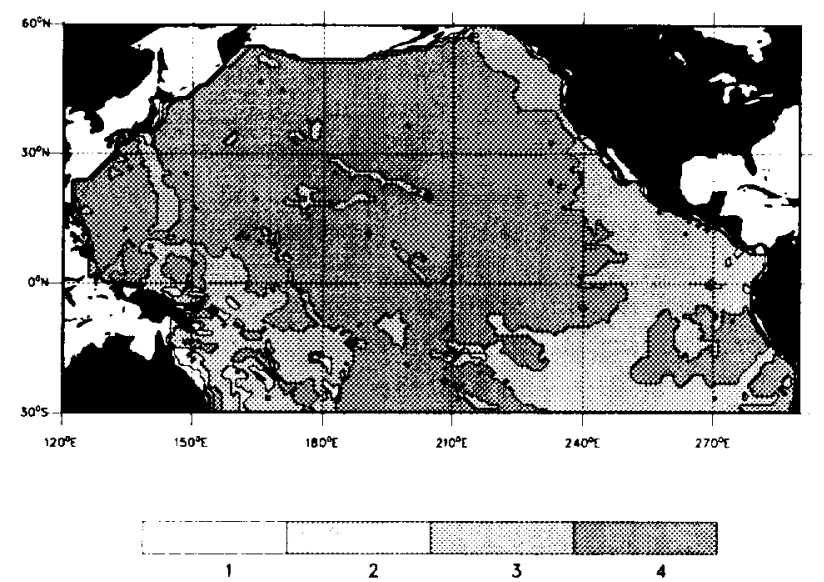

Figure 1a. Domain of the Pacific Ocean model and the number of model layers which reflect the topography used during the estimation studies. Vertical extent of layers is $100,500,1000$, and $2400 \mathrm{~m}$ (from top to bottom), giving a maximum model depth of $4000 \mathrm{~m}$ in the center of the figure.

timescales we are treating, the oceanic response appears to be dominated by near-adiabatic, wave-like motions which are linear. Our fundamental justification is that the method being used appears to "work" in the sense that the principle of superposition applies for scales and amplitudes of the observed differences and for periods up to a year.

We will postulate, in addition, that modeling skill will continue to evolve so that even if some present generation model fails the test of closeness to the data, future models will eventually pass it. (By this means, we achieve a separation of the most immediate problems involved in model construction from those arising in devising estimation procedures.)

The model will be forced to consistency with observations on large scales, and there is an additional assumption that such a model will then also have greater skill in its computation of the smaller scales (e.g., mesoscales) through the scale coupling present in any model, even a linear one. Alternatively, one could force the model

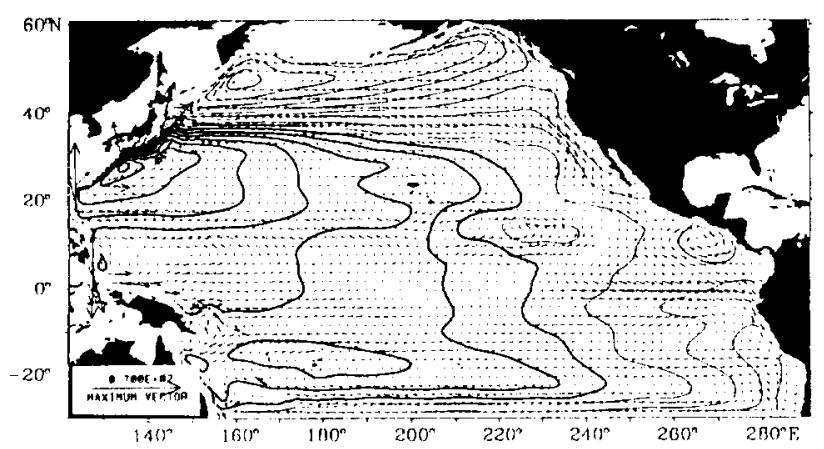

Figure 1b. Sea level elevation (in centimeters) and surface current fields of the model after a 23 -year spinup phase. Every second velocity vector is plotted. Bold and thin lines indicate positive and negative sea level elevation with a contour increment of $10 \mathrm{~cm}$. to consistency with the small scales and anticipate improved skill on the large scale. The actual extent to which OGCMs are controllable through boundary observations confined to restricted ranges of scales is largely unknown. One seeks ultimately to use the altimetry at all scales available from alongtrack data; we confine the present discussion to long wavelengths because it provides an adequate basis to test and demonstrate the method without straining the available computer resources. (There is a resemblance in our strategy to that used by Fukumori and Malanotte-Rizzoli [1995] in a different dynamical setting.)

The paper is organized as follows: The model setup for the Pacific basin is described in section 2. Section 3 discusses the methodology of estimation using altimeter data with pressure Green's functions. Their initialization and successive evolution are discussed in some detail in section 4 . The state estimation method is illustrated in section 5 using synthetic data to provide insight into the potential of reconstructing the oceanic state (three-dimensional flow field and tracer distribution) from surface height/pressure observations. Real $\mathrm{T} / \mathrm{P}$ sea surface height observations are used in section 6 to infer the present-day Pacific circulation and mass distribution.

\section{Model Configuration}

The model used in this study is the primitive equation Geophysical Fluid Dynamics Laboratory (GFDL) model [Bryan, 1969; Cox, 1984], implemented for present purposes in the Pacific Ocean north of $30^{\circ} \mathrm{S}$ with realistic coast lines and bottom topography (Figure 1a). Because the GFDL model is widely used, only those elements necessary to understand the present configuration will be summarized here.

The underlying governing equations which make use of the Boussinesq, hydrostatic, and traditional approximations are conservation equations for momentum, volume, potential temperature $\theta$, and salinity $S$, augmented by a diagnostic equation for density. Bottom and side walls are insulating, i.e., $\left[\partial_{\mathbf{n}}(\theta, S)=0\right]$. A no-slip side wall condition is imposed and the bottom is "free-slip." At the surface there is a rigid lid,

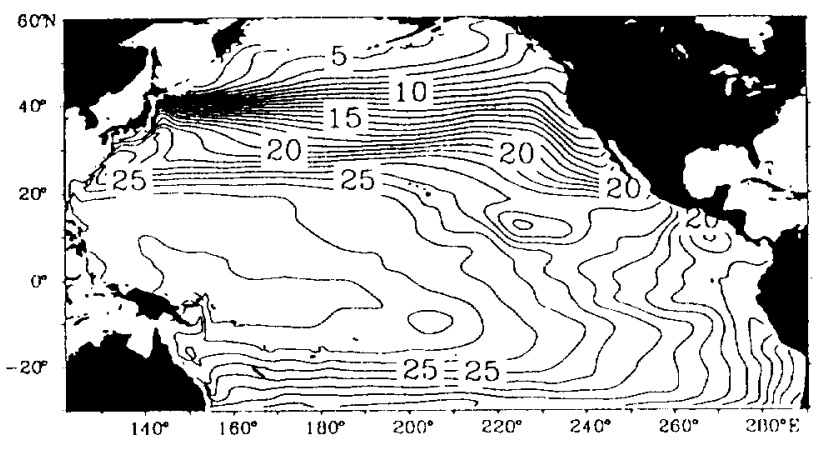

Figure 1c. Surface temperature field corresponding to Figure $1 b$. 

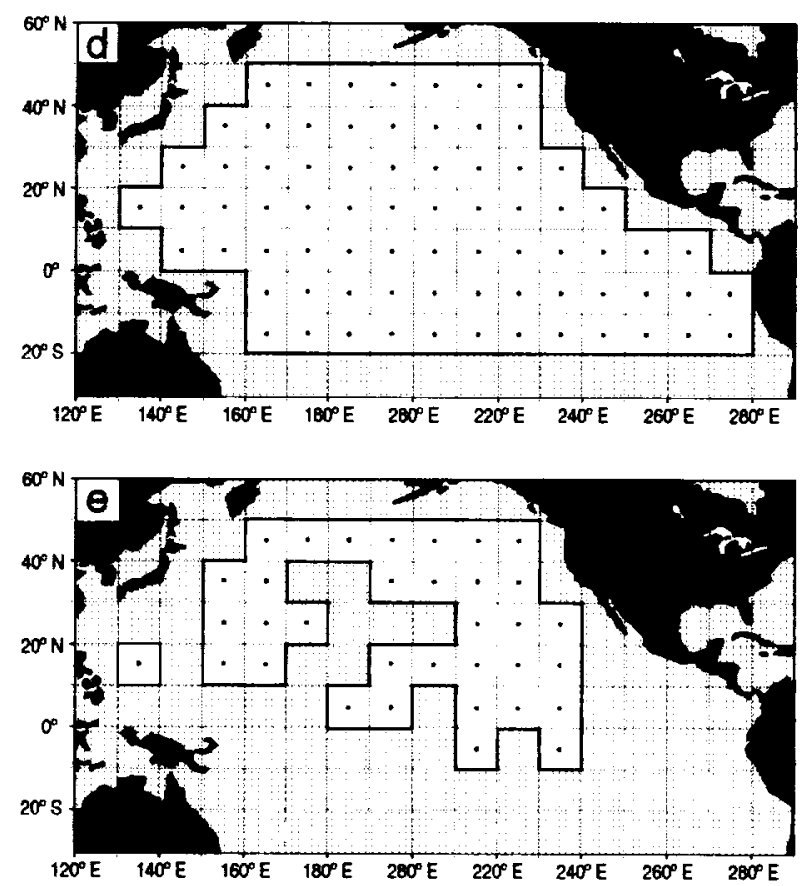

Figure 1d,e. (d) Coarse $10^{\circ}$ by $10^{\circ}$ grid, on which the Green's functions where calculated in layers 1 through 3. (e) Owing to geographical shoaling, the bottom layer has a smaller geographical extent on the coarse grid.

and the momentum flux is given by the wind stress $\left[\partial_{z}(u, v) \propto \tau^{\lambda, \phi}\right]$, with $\lambda, \phi$ being longitude and latitude, respectively. Surface heat and freshwater (salt) fluxes are mimicked by Newtonian damping terms, with the model $\theta$ and $S$ fields relaxed toward Levitus's annual mean surface $\theta_{\mathrm{Lev}}, S_{\mathrm{Lev}}$ fields as $H_{\theta}=\gamma\left(\theta_{\mathrm{Lev}}-\theta\right)$ and $H_{S}=\gamma\left(S_{\mathrm{Lev}}-S\right)$ with timescale $\gamma^{-1}$.

Because our main intention here is the exploration of the method in the context of real data, a limited model resolution is adopted, with a $1^{\circ}$ horizontal grid spacing and with only four layers in the vertical of thickness $100,500,1000$, and $2400 \mathrm{~m}$ (from top to bottom). The maximum model depth is $4000 \mathrm{~m}$. The southern wall and the Indonesian passages are artificially closed for this specific realization. Although this configuration is somewhat limiting in providing a highly realistic model of the circulation of the Pacific, it significantly simplifies the situation without changing the fundamental na- ture of the problem. In future applications, the resolution will be increased substantially, and ultimately, the model will have a global domain.

Starting from Levitus's [1982] annual mean $\theta$ and $S$ distributions and a resting flow field, the model was integrated forward for about 18 years $(160,000$ time steps) with a mean surface wind stress provided by Trenberth et al. [1989] from routine analysis of the European Centre for Medium-Range Weather Forecasts (ECMWF) on a $2.5^{\circ} \times 2.5^{\circ}$ grid and averaged over the period 1980 to 1986. The time step $\Delta t$ was 1 hour, and parameters for mixing and diffusion were used as listed in Table 1. Owing to the relatively strong surface $T$ and $S$ forcing and its damping effects on the surface pressure Green's functions (see below), the relaxation time scale $\gamma^{-1}$ was increased from its initial 30 days to 100 days, and the model was integrated forward another 4.5 years $(40,000$ time steps), giving a total of roughly 23 years of spin-up.

The surface pressure and velocity fields at the end of the 23-year spin-up are shown in Figure $1 \mathrm{~b}$. The related surface temperature field is shown in Figure 1c. In the North Pacific, the major circulation components are simulated. However, because of the low vertical resolution, there is not much resemblance to observations in the tropics, and the circulation south of the equator is dominated by the artificially closed southern boundary.

\section{Methodology}

The estimation procedure introduced below is formulated for observed model/data differences in surface pressure. Our goal is to describe those differences as a solution to linear model dynamics in terms of model Green's functions, using all observations available during a period $\tau$. As explained below in detail, the result is a linear inverse problem for a set of disturbance coefficients, which is then solved. Generally, the linear model can be set up on the full OGCM grid. (To avoid ambiguity, the original model will henceforth be referred to as the "OGCM.") However, consistent with the scale argument given in the introduction, the linear estimation problem is defined on a coarse grid, obtained by dividing the ocean into a series of three-dimensional rectangular boxes of horizontal scale $L=10^{\circ}$ with spatial coverage as shown in Figure 1d. A stack of such coarse grid boxes occupies the whole water column with vertical

Table 1. Model Parameters

\begin{tabular}{lll}
\hline Parameter & Symbol & Value \\
\hline Resolution & $\Delta \phi, \Delta \lambda$ & $1^{\circ}$ \\
$\quad$ Horizontal & $\Delta z$ & $100,500,1000,2400 \mathrm{~m}$ \\
Vertical & $H_{0}$ & $4000 \mathrm{~m}$ \\
Maximum depth & $\Delta t$ & 1 hour \\
Time step & $\lambda^{-1}$ & $30 / 100$ days \\
Relaxation coefficient & $A_{\mathrm{hm}}$ & $10^{4} \mathrm{~m}^{2} \mathrm{~s}^{-1}$ \\
Horizontal mixing & $A_{\mathrm{vm}}$ & $10^{-2} \mathrm{~m}^{2} \mathrm{~s}^{-1}$ \\
Vertical mixing & $A_{\mathrm{hh}}$ & $10^{7} \mathrm{~m}^{2} \mathrm{~s}^{-1}$ \\
Horizontal diffusion & $A_{\mathrm{vh}}$ & $0.5 \times 10^{-4} \mathrm{~m}^{2} \mathrm{~s}^{-1}$ \\
Vertical diffusion &
\end{tabular}


dimensions defined by the layer thickness. Boxes next to the lateral boundaries are not included in the set of perturbed regions because the presence of intense currents and other boundary effects can more readily lead to violations of linearity. The bottom layer (Figure 1e) has a smaller geographical extent owing to topographic shoaling. Only disturbances larger than a horizontal scale $L$ will be examined in this paper. However, in more elaborate applications, this limitation will be relaxed, permitting smaller scales in the estimation process. Ultimately, one needs to take all scales into consideration. The result is a linear model, which is much reduced in dimension from that of the OGCM, both because of the coarser spatial resolution of the former, but also because the linear model physics are now embodied mainly in the (stored) Green's functions rather than in the full model state vector itself. Achieving this state vector dimension reduction is one of the chief goals of the present method.

For notational simplicity, we will apply a one-dimensional counting index to the full three-dimensional grid. In addition, $t=\left\{0,1,2, \cdots, t_{f}\right\}$ denotes a discrete time index with $t_{f}$ defining the period of inversion $\tau_{f}=t_{f} \cdot \Delta t$, and $\Delta t$ being the time step of the linear model. $\Delta t$ can differ from the time step of the OGCM and is chosen here to equal the 10-day sampling period of $\mathrm{T} / \mathrm{P}$. The final time $t_{f}$ corresponds to a 1 -year period of $\mathrm{T} / \mathrm{P}$ data. The sensitivity of the results to the linear model time step and the length of the data period are subject to further investigation.

To establish notation, we write the linear homogeneous model in canonical form as

$$
\mathbf{p}(t+1)=\mathbf{Y}(t) \mathbf{p}(t)
$$

with initial conditions $\mathbf{p}(t=0)=\mathbf{p}_{0}$. Here $\mathbf{r}(\mathbf{t})$ is a matrix operator, and $\mathbf{p}(t)$ is the oceanic state vector at discrete time $t=\{0,1,2, \cdots\}$. Given a pressure Green's function, $\mathrm{G}^{p}\left(t, t^{\prime}\right)$, defined from

$$
\mathbf{G}^{p}\left(t+1, t^{\prime}\right)=\mathbf{\Upsilon}(t) \mathbf{G}^{p}\left(t, t^{\prime}\right),
$$

with initial conditions $\mathbf{G}^{p}\left(t, t^{\prime}\right)=\delta_{t, t^{\prime}}\left(\delta_{t, t^{\prime}}\right.$ being the Kronecker symbol), a solution to (4) can be written as [e.g., Roach, 1982]

$$
\mathbf{p}(t)=\sum_{t^{\prime}} \mathbf{p}_{0}\left(t^{\prime}\right) \mathbf{G}^{p}\left(t, t^{\prime}\right)
$$

We use the superscript $p$, denoting the pressure Green's function, because later we use the associated temperature, salinity, and velocity disturbance fields, to be denoted $\mathbf{G}^{\theta}, \mathbf{G}^{S}$, and $\mathbf{G}^{v}$, respectively.

For the state estimation procedure we make use of (4)-(6). For this purpose, a linearized version of the fully nonlinear model is derived empirically, by disturbing the spun-up, and forced OGCM by a small perturbation. The subsequent model evolution (over a 1-year period) relative to a similar, but unperturbed, parallel run defines the linear model according to (4), with the state vector $\mathbf{p}(t)$ denoting the difference in the states of the two parallel OGCM runs. Accordingly, we derive the impulse response function, $G_{i, j}^{p}\left(t, t^{\prime}\right)$, of the linearized model as the time-evolving response of the perturbed OGCM relative to an unperturbed parallel run, at any grid point $i$ of the full three-dimensional OGCM grid and at discrete time $t$, with a unit amplitude pressure anomaly imposed at grid point $j$ and at $t=t^{\prime}$. By definition, $G_{j j}^{p}\left(t^{\prime}, t^{\prime}\right)=1$, and

$$
G_{i, j}^{p}\left(t, t^{\prime}\right)=0, t<t^{\prime},
$$

the latter condition stating causality, i.e., the model will not have responded to future perturbations. Here and below, the indices $i$ denote the grid points where a response is observed, while the $j$ grid corresponds to points at which perturbations are permitted. (There is some ambiguity introduced by our use of the label "Green's functions." We do not expect our empirical linear model to be self-adjoint, and for such systems a second set of, adjoint, Green's functions can also be defined [Morse and Feshbach, 1953]. Our definition of $G_{i, j}^{p}\left(t, t^{\prime}\right)$ is that of a unit impulse response function for the linear model itself as defined in (6).)

Note that the linear model is obtained here through a "black-box" procedure, in which the impulse responses are found empirically, assuming that the OGCM will respond in a linear fashion to small, local perturbations. However, we also will make use of the simplifying assumption that the Green's functions are translation invariant in time, that is, $\mathbf{G}^{p}\left(t, t^{\prime}\right)=\mathbf{G}^{p}\left(t-t^{\prime}, 0\right)$. If the model basic state changes significantly, e.g., if there are large seasonal fluctuations, this assumption will fail, and we would be forced to compute different Green's functions for different seasons. The major drawback to such a need would be greatly increased storage requirements. Within the limits of the available altimeter data, we have found no indication that this refinement is necessary.

We assume now that Green's functions $\left\{G_{i, j}^{p}\left(t, t^{\prime}\right)\right\}$ have been determined for a complete set of $j$ points, $j=\{1,2, \cdots, J\}$, and shift to the estimation problem. In the present context of altimetric sea surface height data, observations are restricted to grid points residing in the coarse resolution surface layer $S \subset B$, where $S$ denotes grid points in the surface layer and $B$ is the three-dimensional grid. Then

$$
\tilde{\delta p_{i}}(t)=\left(g \rho \zeta_{i}(t)-\tilde{p}_{i}(t)\right) \quad i \in S
$$

defines the difference in surface pressure between the OGCM first estimate $\tilde{p}_{i}(t)$ and the observed value $g \rho \zeta_{i}(t)$. The first estimate $\tilde{p}_{i}(t)$ is obtained by integrating the OGCM forward in time starting from the given initial state with prescribed boundary conditions.

In the absence of any of the errors discussed below, the $\delta p_{i}(t)$ defines the "observations" for our linearized estimation problem. However, in practice, there are always errors present, and instead the observations must be written as

$$
y_{i}(t)=\tilde{\delta p_{i}}(t)+n_{i}(t) .
$$


According to the definition of $\tilde{\delta p}_{i}(t)$, the term $n_{i}(t)$ arises from errors in both the data and the model (see below). We seek to find the best estimates $\hat{\alpha}$ of the true forcing coefficients $\alpha$ and their corresponding uncertainties $P_{\alpha, \alpha}$ (see $(20)$ ), which lead to a best fit $\hat{\delta p}$ of the true $\delta \mathbf{p}$ in an optimal sense,

$$
\begin{aligned}
\hat{\delta p_{i}}(t) & =\sum_{j=1}^{J} \sum_{t^{\prime}=0}^{t_{f}} \hat{\alpha}_{j}\left(t^{\prime}\right) G_{i, j}^{p}\left(t, t^{\prime}\right) \\
& =\sum_{j=1}^{J} \sum_{t^{\prime}=0}^{t_{f}} \hat{\alpha}_{j}\left(t^{\prime}\right) G_{i, j}^{p}\left(t-t^{\prime}, 0\right)
\end{aligned}
$$

plus an estimate of the yet to be determined residual (noise) term $\hat{n}_{i}(t)$. This state estimation process can be interpreted as the projection of the noisy "observations" onto the dynamical set of linear basis functions: the Green's functions. In (10), the second equality follows from translation invariance in time. At the final time step $t_{f}$, the convolution (10) is carried over the entire period for which data are available. However, if a disturbance in the ocean were fully dissipated after, e.g., 50 days, then $t_{f}$ could be reduced, accordingly. In our application, $t_{f}$ is assumed to be the whole 1-year period of $\mathrm{T} / \mathrm{P}$ data.

An estimate $\hat{p}_{i}(t)$ of the true absolute pressure $p_{i}(t)$ at any location of the full model domain $i$ and at time step $t$, consistent with altimetric surface observations, is then obtained from the sum

$$
\hat{p}_{i}(t)=\tilde{p}_{i}(t)+\hat{\delta} p_{i}(t) .
$$

We pause here to introduce an example: suppose, hypothetically, the OGCM predicts the correct pressure at $t=0$ and subsequently everywhere in the model domain, except for the contribution owing to an unknown perturbation at grid point $j_{0}$ at $t=0$, which subsequently propagates through the model area. Such a perturbation could, e.g., be associated with an error in the wind stress field, used to derive the OGCM. Then determination of $\alpha_{j_{0}}(0)$ properly initializes the model, while all $\alpha_{j_{0}}\left(t^{\prime}\right), t^{\prime}>0$, and all $\alpha_{j}\left(t^{\prime}\right), j \neq j_{0}$, at all $t^{\prime}$, vanish. In this special case, (10) would read

$$
\hat{\delta p_{i}}(t)=\hat{\alpha}_{j_{0}}(0) G_{i j_{0}}^{p}(t, 0)
$$

which provides a perfect estimate of the anomaly everywhere for all future times, and the sum (11) would reproduce the observations with no further correction required. A further unpredicted disturbance at the same grid point $j_{0}$ and $t=1$ would require the determination of the two unknowns $\hat{\alpha}_{j_{0}}(0), \hat{\alpha}_{j_{0}}(1)$, leading to

$$
\hat{\delta p_{i}(t)}=\hat{\alpha}_{j_{0}}(0) G_{i j_{0}}^{p}(t, 0)+\hat{\alpha}_{j_{0}}(1) G_{i j_{0}}^{p}(t, 1) .
$$

In general, one solves simultaneously for all $\hat{\alpha}_{j}\left(t^{\prime}\right)$ using all the observations, and summing over all $j$ produces the general linearized response (10) at grid point $i \in B$. The collection $\left\{\alpha_{j}\left(t^{\prime}\right)\right\}$ represents the state vector of the linear model. The assumption of linear dynamics made in (8)-(10) is that disturbances are superposable to adequate accuracy.

The process of model correction in (10) can be thought of as one of constantly reinitializing the linear model to the extent that unpredictable forces act, or disturbances propagate into the estimation domain. The determination of the required perturbation values (corrections) is made in such a manner that the resulting model trajectory best fits the observations over the entire data period and model domain in a way consistent with the linear dynamics inherent in the Green's functions. This procedure contrasts with the so-called "blending technique" [e.g., Ghil and Malanotte-Rizzoli, 1991], by which models are re-initialized by direct insertion of the most recent observations.

In our context, $\mathrm{T} / \mathrm{P}$ observations are prescribed only on the coarse grid in the surface layer $i=\{1, \cdots, I\}$. To render the estimation problem at its simplest level, $j$ could similarly be confined to the coarse grid surface layer, i.e., the special case in which external disturbances are confined to the surface. In either case, the collection of observations over the complete data time span form a set of simultaneous equations for the unknown coefficients $\boldsymbol{\alpha}=\left\{\alpha_{j}\left(t^{\prime}\right)\right\}$ :

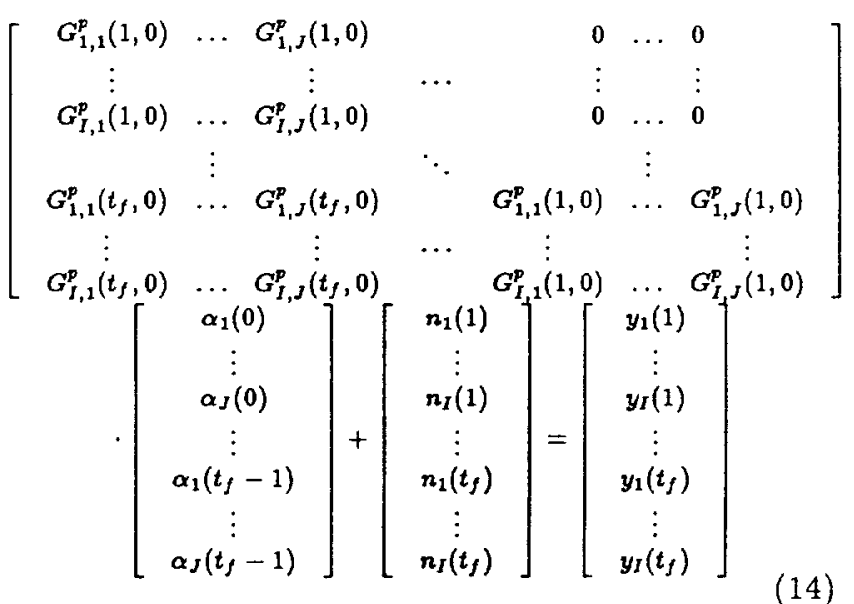

In matrix form, (14) is

$$
\mathbf{G}^{p} \boldsymbol{\alpha}+\mathbf{n}=\mathbf{y}
$$

where $\mathbf{n}$ represents the noise in all positions at all times and $y$ respresents the collection of observations. However, more generally, one must accommodate disturbances at depth, which can arise both from initialization errors and from errors propagating into the estimation domain. Then $(10)$ is generalized to having the $j$ grid distributed over the full three-dimensional domain with the surface observations being a summation of contributions from perturbations occurring in all model layers. The Green's function $G_{i, j}^{p}\left(t, t^{\prime}\right)$ is then the response observed in the surface layer at grid point $i$ due to a pressure disturbance imposed at time step $t^{\prime}$ at any surface or subsurface location.

In an equivalent formulation, which provides further insight into the vertical structures giving rise to observed surface perturbations, one would rewrite each 
Green's function as a set of vertical basis functions. Suppose, for example, that the ocean has a flat bottom. Then the linear dynamical modes $m=\{0,1,2, . . M\}$ would provide a natural choice of a vertical basis. Disturbances at point $i$ would then be composed of perturbations by mode $m$, and resulting anomalies $\delta \hat{\mathbf{p}}^{m}(t)$ would be the history of the pressure anomalies of each mode $m$. Empirical orthogonal functions (EOFs) or any other basis could be used similarly. However, because linear dynamical modes in the presence of varying bathymetry are defined only locally, we use formulation (15), in which each of the coefficients excites energy in the vertical modes. Including the subsurface coefficients permits one to construct the equivalent of modes, either barotropic or baroclinic.

The system is a linear time-evolving one, and there are several options for determining the state vector $\hat{\alpha}$. To render the discussion simple, we will consider a solution obtained by the method of singular value decomposition (SVD [e.g., Wunsch, 1996]) which uses the entire domain of observations all at once. If (15) becomes too large, sequential methods, such as filters and smoothers, or recursive methods can be employed. Application of these standard approaches would normally lead one to use the canonical form (4) ( $\boldsymbol{\Upsilon}$ is readily determined from $\mathbf{G}^{p}$ by (5) [see Menemenlis and Wunsch, 1996]).

We solve (15) after column scaling, written as

$$
\mathbf{G}^{p} \mathbf{W}^{-\frac{1}{2}} \mathbf{W}^{\frac{1}{2}} \boldsymbol{\alpha}+\mathbf{n}=\mathbf{y},
$$

where $\mathbf{W}$ has the diagonal form

$$
\begin{gathered}
\mathbf{W}=\operatorname{diag}\left\{\left(\cdots h_{1} / H_{0} \cdots\right),\left(\cdots h_{2} / H_{0} \cdots\right),\right. \\
\left.\left(\cdots h_{3} / H_{0} \cdots\right),\left(\cdots h_{4} / H_{0} \cdots\right)\right\},
\end{gathered}
$$

with $h_{i}$ denoting the layer thickness of the $i$ th layer and $H_{0}$ is the total depth. Let the SVD of the Green's function matrix $\mathbf{G}^{p} \mathbf{W}^{-\frac{1}{2}}$ be

$$
\mathbf{G}^{p} \mathbf{W}^{-\frac{2}{2}}=\mathbf{U} \boldsymbol{\Lambda} \mathbf{V}^{T},
$$

with $\mathbf{U}$ and $\mathbf{V}$ containing the left and right singular vectors, $\boldsymbol{\Lambda}$ having the singular values on its diagonal, and the superscript $T$ denoting the transpose. Then the estimated state vector $\hat{\alpha}$ is

$$
\hat{\boldsymbol{\alpha}}=\mathbf{W}^{-\frac{1}{2}} \mathbf{V}_{K} \boldsymbol{\Lambda}_{K}^{-1} \mathbf{U}_{K}^{T} \mathbf{y}
$$

with an estimated uncertainty

$$
\begin{aligned}
\mathbf{P}_{\alpha \alpha} & =<(\hat{\boldsymbol{\alpha}}-\boldsymbol{\alpha})(\hat{\boldsymbol{\alpha}}-\boldsymbol{\alpha})^{T}> \\
& =\sigma^{2} \mathbf{W}^{-\frac{2}{2}}\left(\mathbf{V}_{K} \boldsymbol{\Lambda}_{K}^{-2} \mathbf{V}_{K}^{T}+\mathbf{Q}_{v} \mathbf{R}_{\alpha \alpha} \mathbf{Q}_{v}^{T}\right) \mathbf{W}^{-\frac{T}{2}}
\end{aligned}
$$

distinguishing the true $\boldsymbol{\alpha}$ from the estimated one $\hat{\boldsymbol{\alpha}}$. Here $K$ is the rank of the system and is determined in practice by prior assumptions about the white noise variance $\sigma^{2}$ and the magnitude of the singular values $\lambda_{i}$ (not to be confused with longitude $\lambda$ ); the noise is discussed further below. Column vectors of $\mathbf{Q}_{v}$ are the null space vectors, $\mathbf{R}_{\alpha \alpha}$ is the second moment matrix of the null space coefficients, and $P_{\alpha a}$ is the sum of the noise uncertainty and the uncertainty from the null space variance. Information about $\mathbf{R}_{\alpha \alpha}$ must be provided from prior information; in its absence, one can speak only of the unresolved components. See Wunsch [1996] for further details.

Given estimated coefficients $\hat{\boldsymbol{\alpha}}$, the linear model variables are obtained on the full three-dimensional grid, $i \in B$, from equations like (10) as a function of time, with

$$
\begin{aligned}
& \hat{\delta p_{i}}(t)=\sum_{j}^{J} \sum_{t^{\prime}=0}^{t_{j}} \hat{\alpha}_{j}\left(t^{\prime}\right) G_{i, j}^{p}\left(t-t^{\prime}, 0\right) \\
& {\left[\hat{\delta \theta_{i}}, \hat{\delta S_{i}}, \hat{\delta \mathbf{v}_{i}}\right](t)=\sum_{j}^{J} \sum_{t^{\prime}=0}^{t_{f}} \hat{\alpha}_{j}\left(t^{\prime}\right) G_{i, j}^{[\theta, S, \mathbf{v}]}\left(t-t^{\prime}, 0\right)}
\end{aligned}
$$

where the matrices $\mathbf{G}^{[\theta, S, \mathbf{v}]}$ describe the response of the temperature, salinity, and velocity fields to the associated pressure perturbation. The velocity field also follows readily from the resulting pressure field by geostrophy, and in practice, $\delta \hat{v}_{i}(t)$ was found this way.

Uncertainties of the estimated fields are obtained from

$$
\begin{gathered}
\mathbf{P}_{p p}=\mathbf{G}^{p} \mathbf{P}_{\alpha \alpha} \mathbf{G}^{p T} \\
\mathbf{P}_{[\theta, S, \mathbf{v}]}=\mathbf{G}^{[\theta, S, \mathbf{v}]} \mathbf{P}_{\alpha \alpha} \mathbf{G}^{[\theta, S, \mathbf{v}]^{T}}
\end{gathered}
$$

A few additional comments seem to be useful. First, the linear model trajectory is taken about the prior estimate OGCM state in an assumption essentially the same as used in what is known as the "linearized Kalman filter" [see Wunsch, 1996]. A generalization would be to linearize about a new state (the OGCM plus linear model state) which in the sequential estimation context, leads to the "extended Kalman filter" and associated smoothers. There is a parallel set of linearizations used in adjoint/Pontryagin principle methods in which the adjoint model represents a linearization about the instantaneous OGCM state, which will change through the iterative solution used in that method. There is also a close connection of the present method with that of "representers" (see Bennett [1992] for details). As with any filter-smoother approach, (14) uses observations from the entire estimation period to determine the estimated state at any specific time. This can be understood by recognizing, e.g., that $\boldsymbol{\alpha}(0)$ is multiplied by $\mathbf{G}(N, 0)$ to couple future observations with estimates at prior times.

No attempt has been made here to evaluate directly the relative numerical efficiencies of the present "wholedomain" approach relative to that of a sequential or other solution method (although, in general, whole domain methods, because they do not require multiple computations of the error covariance as filters or smoothers do, are far more efficient). Our focus is on demonstrating the concept of numerical linearization as a workable methodology. In general terms, however, the Green's function approach can be thought of as being related to an inverse operator which is vastly reduced in dimensions to contain only that kernel required by the specific data type and distribution: the surface pressure 
boundary condition. In contrast, the adjoint operator of the (linearized) model would need to invert the complete model state in space and time.

It is possible that when Green's functions solutions are computed with high resolution, numerical pathologies will arise requiring spatial and/or temporal smoothing, but we do not yet have experience with this situation.

Because observations are taken here to be model/data differences, the "noise" $n_{i}(t)$ represents all the errors of the perturbation pressures at the surface, including remaining environmental or geophysical data errors from such sources as tides and orbits, and errors due to missing model physics and insufficient forcing fields and for processes on scales smaller than the dimension of the Green's function representation areas. We will generally assume that

$$
\begin{gathered}
<\mathbf{n}(t)>=\mathbf{0},<\mathbf{n}(t) \mathbf{n}^{T}(t)>=\mathbf{R}(t), \\
<\mathbf{n}(t) \mathbf{n}^{T}\left(t^{\prime}\right)>=\mathbf{0}, t \neq t^{\prime} .
\end{gathered}
$$

The determination of the actual noise level and structure must be addressed using real data. Systematic model errors will not be adequately represented through the joint covariance $\mathbf{R}$, nor will systematic (geographically correlated, time-independent) errors in the $\mathrm{T} / \mathrm{P}$ observations. Careful posterior tests are required to test the assumptions. Uncertainties in $\mathbf{G}^{p}$ are also present owing to the initialization procedure and the subsampling scheme. Discussion of the influence of such errors on the results is a nonlinear problem (sometimes called "total leastsquares" [e.g., van Huffel and Vandewalle, 1991; Wunsch, 1996]). We will ignore such errors in this present discussion.

\section{Finding Green's Functions}

Conceptually, we seek to determine the response of the spun-up OGCM to an initial, regionalized, and weak, surface elevation/pressure perturbation

$$
\zeta(\phi, \lambda)=\zeta_{0} \delta_{\mathbf{r}, \mathbf{r}_{0}} \delta_{t, t_{0}}
$$

confined to a single surface grid point at the geographical location $\mathbf{r}_{0}=\left(\phi_{0}, \lambda_{0}\right)$ in the surface layer. However, with a rigid lid, the surface elevation is not part of the OGCM state vector, and we cannot directly perturb the model with a surface pressure perturbation. To within the quasi-geostrophic approximation, however, the response to an initial geostrophically balanced vortex,

$$
\mathbf{v}=\frac{g}{f}(\hat{\mathbf{k}} \times \nabla \zeta) e^{-\left(\frac{t-t_{0}}{\tau}\right)^{2}}
$$

again confined to a single surface point as $\zeta(\phi, \lambda)=$ $\zeta_{0} \exp \left\{-\left[\left(\lambda-\lambda_{0}\right)^{2}+\left(\phi-\phi_{0}\right)^{2}\right] / L^{2}\right\} \quad$ (Figure 2), is equivalent to imposing a pressure perturbation on the model. The spacescales and timescales are extended to $L=5^{\circ}$ and $\tau=5$ days to represent an initial pulse-like perturbation after its geostrophic adjustment process on a rotating sphere. The general problem of geostrophic adjustment has a large literature going back

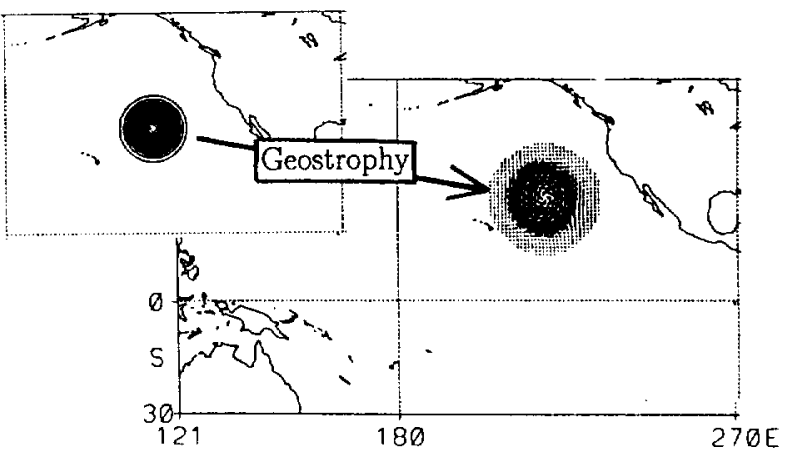

Figure 2. Schematic of the model perturbation. An initial height disturbance is transformed into a geostrophically balanced vortex, which is imposed on the model surface layer. See text for details on spacescales and timescales of the initialization procedure.

at least to Hough [1897], and including the famous paper of Rossby [1936]. Blumen [1972] and Gill [1982] provide comprehensive reviews. From those studies, an initial adjustment timescale of the order of several in. ertial periods is found.

Green's functions were computed and stored for initial disturbance (27) in each of the coarse grid points. The linear model pressure Green's function $\mathbf{G}^{p}$ is obtained by the normalization of the resulting time series of the pressure perturbation by its maximum value (resulting in $\left.G_{j, j}^{p}(0)=1\right)$. The matrices $\mathbf{G}^{[\theta, S, \mathbf{v}]}$ are obtained from the related temperature, salinity, and velocity fields.

The adjustment process of the interior ocean to surface pressure disturbances is of interest in its own right. (One must distinguish perturbations within the uppermost layer of the ocean from those imposed as atmospheric loads; for the latter, see Ponte, [1992], and Wunsch and Stammer, [1996].) We confine the discussion here to a brief summary of the response as obtained from the numerical model. Analytical solutions of relevance to our computations can be found in the works by Bolin [1953], Fjeldstad [1957], Longuet-Higgins [1965], Geisler [1970], Philander [1978] and Blumen [1972]. In a different context, a related model response obtained from a subsurface density perturbation was analyzed by Sarmiento and Bryan [1982], which in its mass transport stream function shows a wavelike pattern in the central North Atlantic, very similar to the results discussed below.

The response of the model to a localized surface pressure disturbance associated with the isolated vortex (27) centered at $35^{\circ} \mathrm{N}, 220^{\circ} \mathrm{E}$ is depicted in Figure 3 in terms of its surface pressure and flow field after $t=16$ and $t=41$ days (from now on, we use $t$ as dimensional time). An expected asymmetric pattern of a radiating Rossby wave field is visible, which rapidly spreads over the entire basin and reaches the Kuroshio within about 10 days. The dominant wavelength decreases with time, as the longer waves are reflected and dissipated. After about 50 days, the barotropic wave field is largely gone. The influence of topographic features such as 

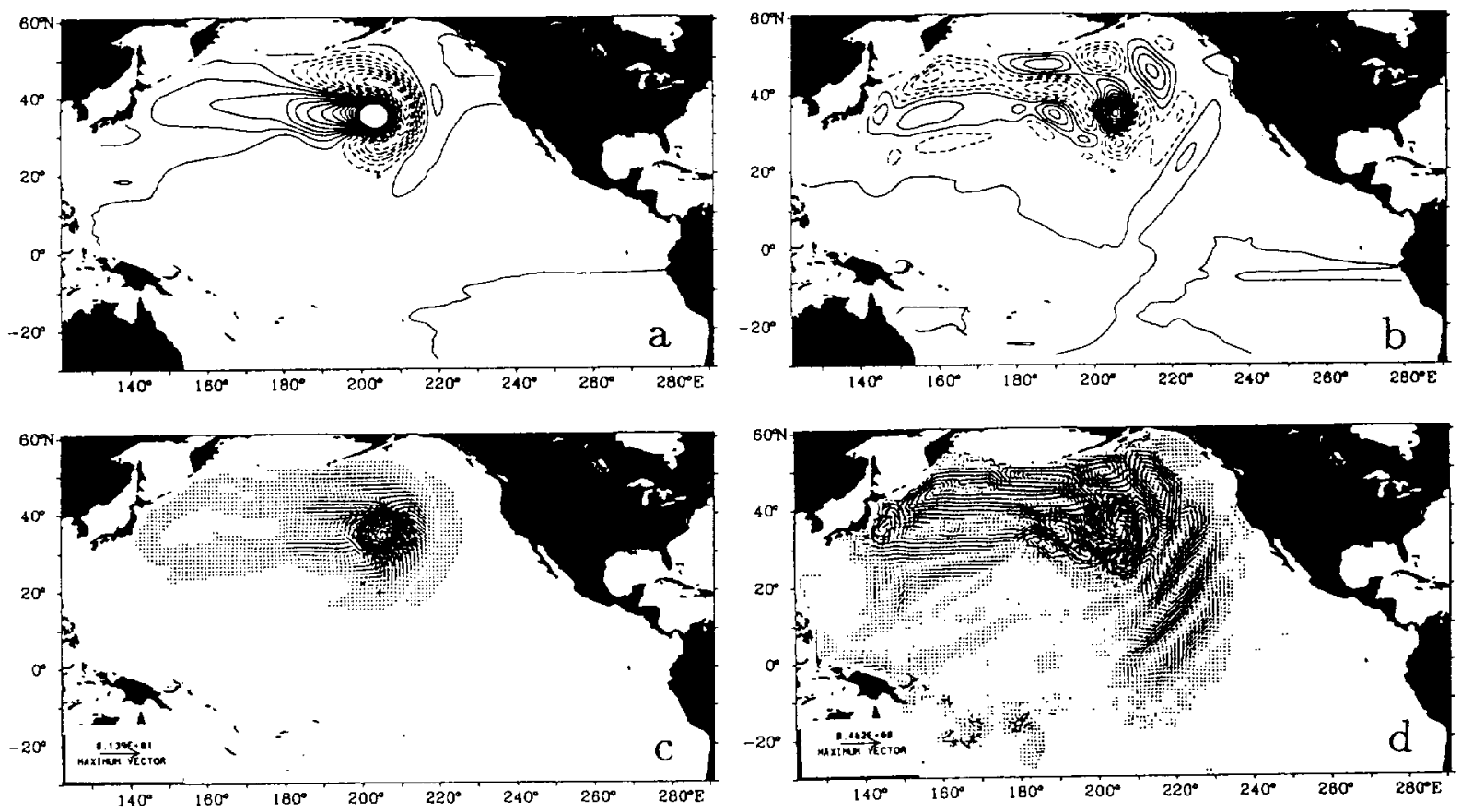

Figure 3. A Green's function, illustrated from the surface pressure and velocity fields (top and bottom panels, respectively) at $t=16$ (left) and $t=41$ days (right). The initial perturbation was imposed at $30^{\circ} \mathrm{N}, 215^{\circ} \mathrm{E}$. Note the pronounced influence of the bathymetric features on the propagation characteristics of the developing fast Rossby wave field. Maximum values are clipped in Figure 3a. The contour increment is $0.1 \mathrm{~cm}$.
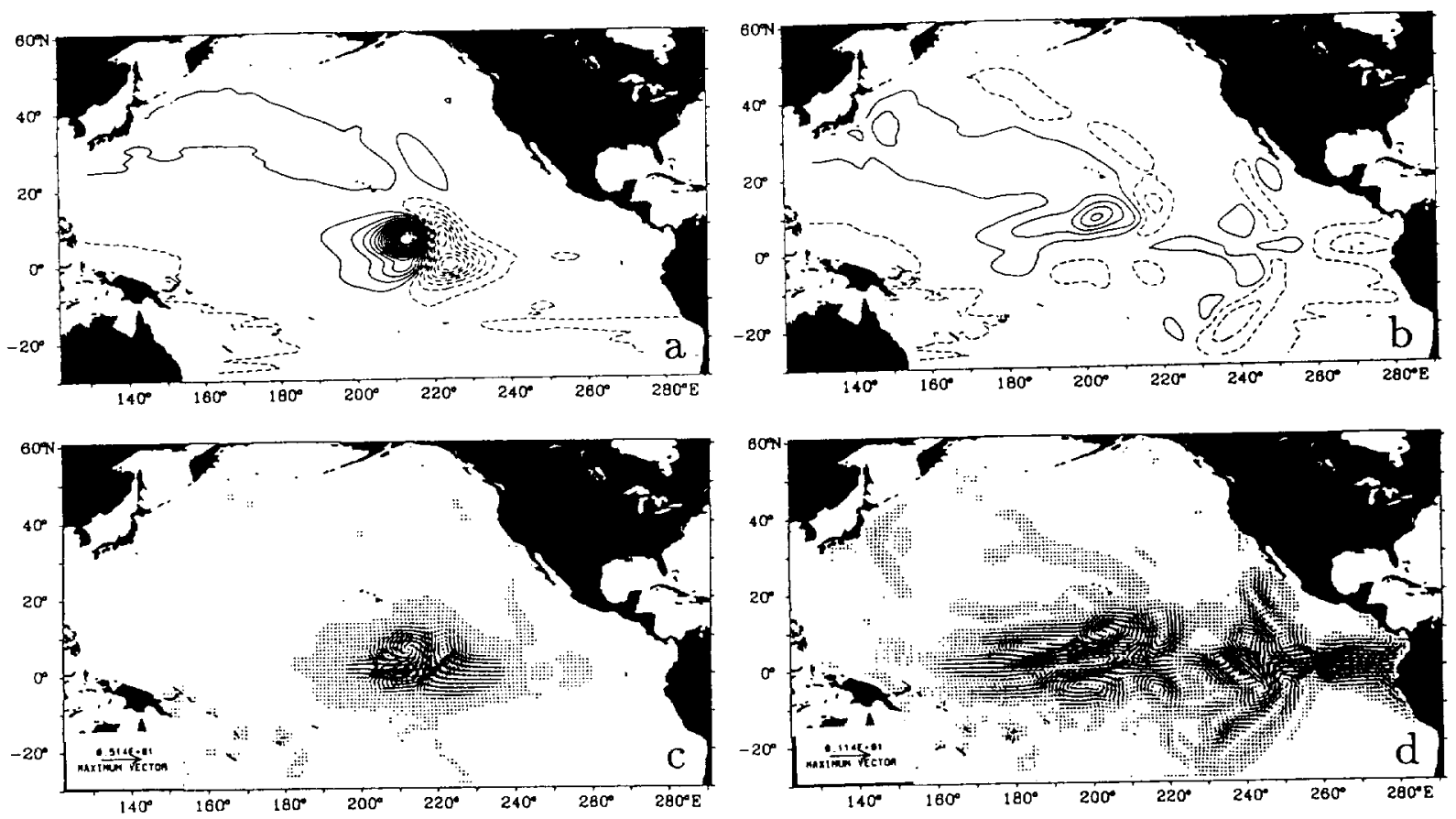

Figure 4. Same as Figure 3 , but for a perturbation imposed at $5^{\circ} \mathrm{N}, 215^{\circ} \mathrm{W}$ and subsampled at $t=25$ days (left) and $t=58$ days (right). Unlike the perturbation in midlatitudes, the enhancement of a low-mode Kelvin wave at the equator and its reflection at the eastern side is visible. The contour increment is $0.1 \mathrm{~cm}$. 
the Hawaiian Ridge or the Emperor Seamount Chain is apparent in Figure $3 \mathrm{~b}$, effectively blocking the fast barotropic waves.

The model response, which in midlatitudes shows predominantly westward propagation of locally imposed signals, is altered in the tropics: a velocity anomaly imposed at $5^{\circ} \mathrm{N}, 215^{\circ} \mathrm{E}$ (Figure 4) generates a fast eastward moving low-mode baroclinic Kelvin wave along with a westward going baroclinic equatorial Rossby wave. The Kelvin wave is partially scattered by the East Pacific Rise before reaching the eastern boundary. In agreement with the theory of equatorial dynamics [e.g., Philander, 1978], the low-latitude disturbance, being predominantly baroclinic in vertical structure, spreads zonally but does not penetrate far into high latitudes.

In the vertical, the model response is generally a sum of all dynamical modes (the barotropic plus three baroclinic) which are excited by the initial disturbance. Figure 5 depicts a zonal section of the meridional velocity component through the center of the initial disturbance over a 1-year period. As can be seen from the figure, the fast initial barotropic response is followed by the emergence of increasingly high baroclinic modes, each of which moves westward with its associated Rossby wave phase speed. The anticyclonic vortex imposed initially only in the surface layer induces an ageostrophic flow component into the vortex center and an associated secondary vertical velocity field (not shown) with downwelling in the vortex interior and upwelling at the outer edges which is associated with the return flow in the vertical. The consequent shift of the isotherms leads to a warming in the vortex center and a cooling at its outer edges. The complex interior response is important in a general sense: surface elevation/pressure is coupled to interior motions over the entire water column, motions which we can hope to infer from an inverse computation.

When a perturbation is introduced in a subsurface layer, a number of expected changes can be observed. Figure 6 shows the response to a perturbation in either of the four layers at $35^{\circ} \mathrm{N}, 205^{\circ} \mathrm{E}$ after 83 days. The remote barotropic field is largely independent of the specific depth of the initial disturbance. In the nearfield, the details of the baroclinic response are different. Consistent with the dominance of the first baroclinic mode, the immediately overlying surface response to a pressure disturbance in layers 3 and 4 is of opposite sign, in contrast to a perturbation imposed in the two surface layers. In addition, details of the energy partitioning of baroclinic modes depends on the depth of the initial perturbation (e.g., giving rise to enhanced second-mode energy in low latitudes when initialized at middepth).

\section{A Twin Experiment}

We now turn to the estimation problem: to calculate the full three-dimensional oceanic state at all times of interest as outlined in section 3 , given only altimetric observations of the sea surface elevation field (or equivalently, the related geostrophic flow in the surface layer) and a model forecast.
Although our primary interest is in the results from actual data, we nonetheless will digress briefly to describe the results obtained with perfect "data" from a so-called twin experiment. Real observations raise questions which are rarely encountered in discussions of estimation with artificial data, but use of artificial data permits us to isolate problems with the method which are independent of the complexities of real observation uncertainties. The purpose of this intermediate step is to establish the estimation problem in the presence of a completely known ocean state. Because of the absence of good in situ data coverage simultaneous with the altimetry, a twin experiment is the only basis for exploring a comparison over the full depth range.

Starting from the spun-up state, the OGCM was integrated forward for one additional year, the time history

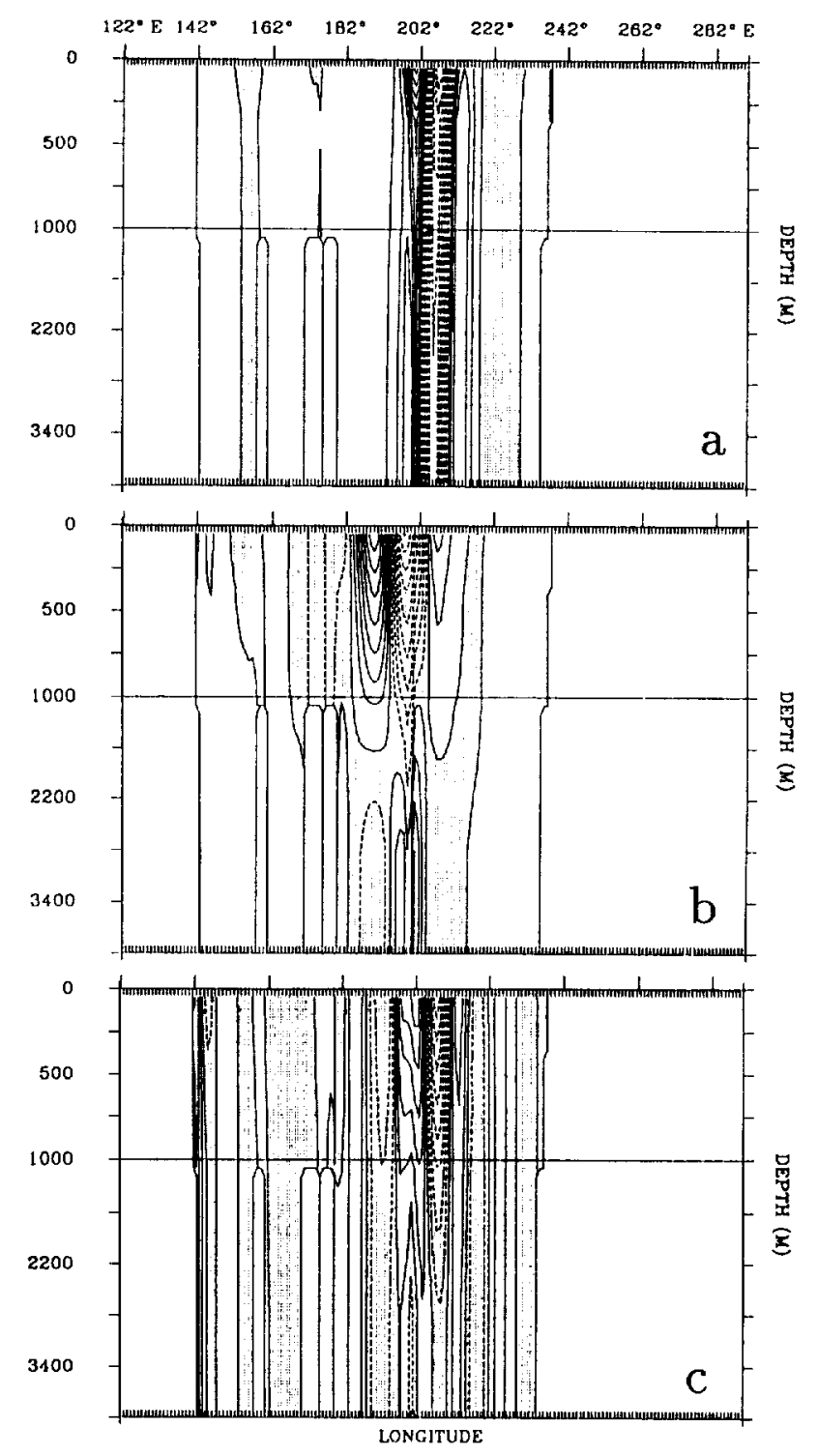

Figure 5. Zonal sections of the northward $(v)$ velocity component (corresponding to Figure 3 ) through the center of the initial velocity perturbation for (a) $t=16$, (b) 41 , and (c) 410 days. Negative values are stippled. An arbitrary contour increment is shown, to indicate vertical structures. 

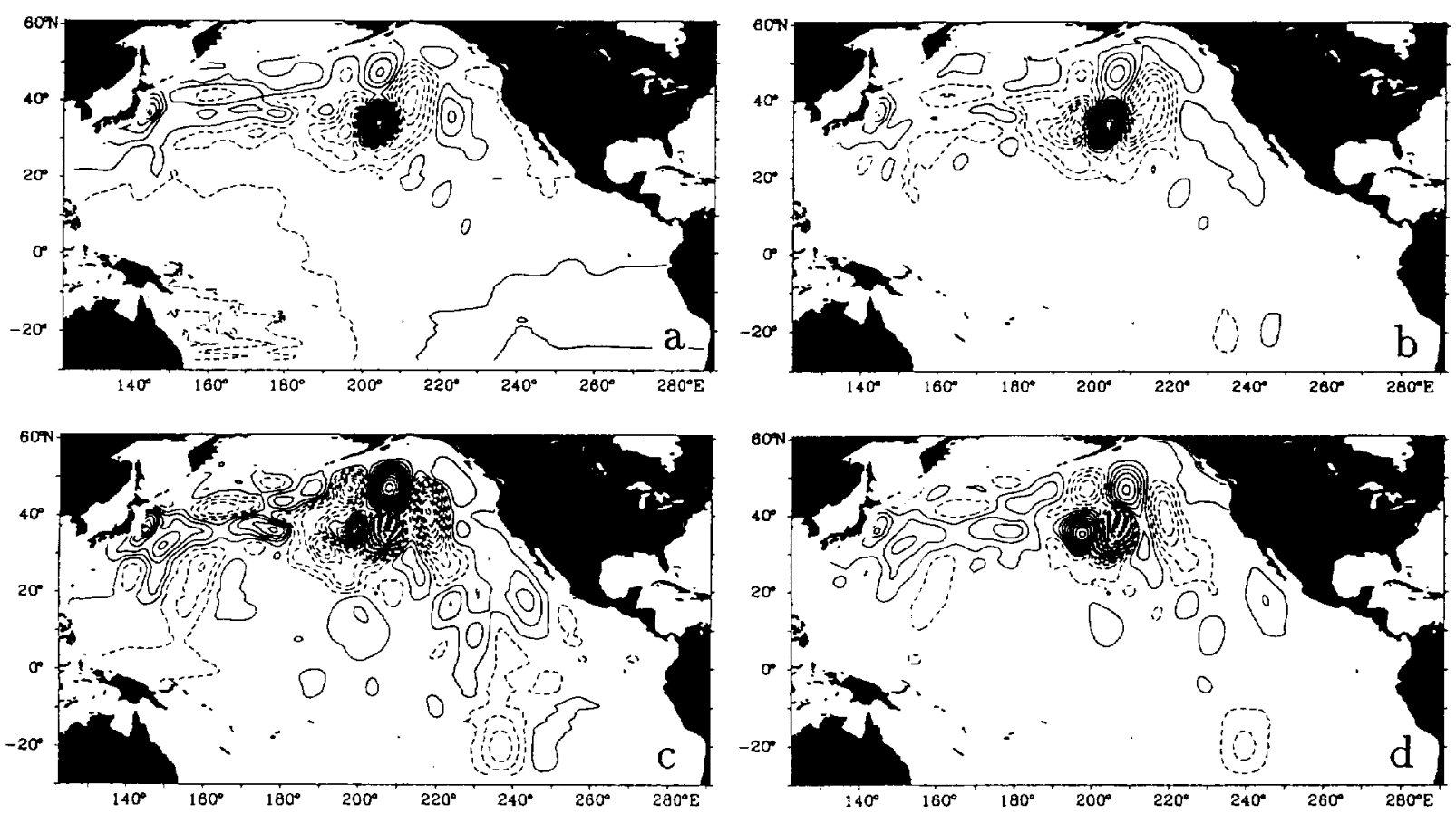

Figure 6. Sea surface pressure fields, which result from putting velocity perturbations into layer (a) 1 , (b) 2, (c) 3 and (d) 4, after 83 days. The contour increment is $0.05 \mathrm{~cm}$.

of which gave the first-estimate reference state. To generate artificial "model data," the OGCM forcing was changed from constant to monthly mean wind stress values, and run for an additional 10 years into a new climatological equilibrium. The subsequent eleventh year was taken as the "true" state, which is supposed to be recovered from the surface "observations," in our framework, taken to be the surface elevation anomalies referenced to the previous reference state. As an example, Figure 7 shows instantaneous anomaly fields of surface pressure and velocity, representing winter conditions. Such anomaly fields were sampled on the coarse grid every 10 days over a 1 -year period to form the vector of observations $\mathbf{y}$ as stated in (14).

To obtain more insight into the problem of estimating the full ocean state from surface height/velocity obser-

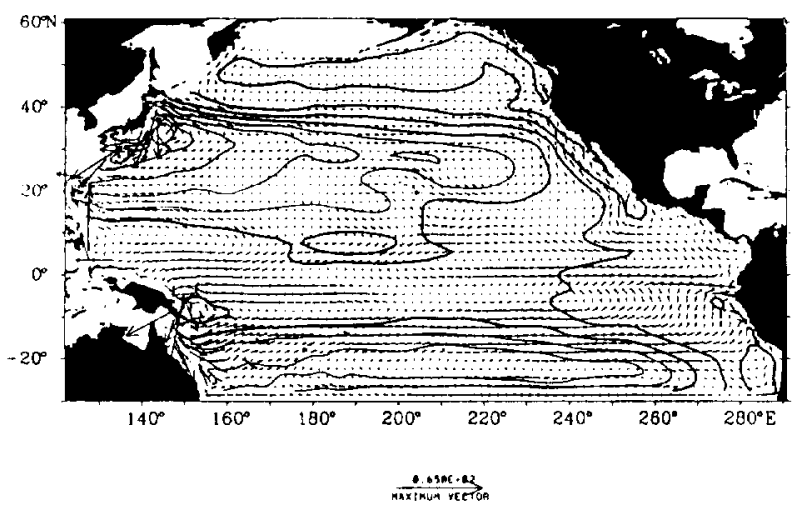

Figure 7. Instantaneous difference fields of $p_{s}$ and horizontal surface velocity $\mathbf{v}$ between the synthetic "model data" and the OGCM first guess, representing winter conditions. The contour increment is $10 \mathrm{~cm}$. vations, various experiments were performed, all listed in Table 2. In a first experiment, labeled G1, both observations and coefficients $\alpha$ are confined to the coarse grid in the surface layer only. There are then 2590 unknown values ( 74 points times 35 time steps of 10 days each), and an equal number of observations connected by (14). Confining the unknowns to the surface layer is equivalent to the assumption that only in the surface layer do unpredictable changes take place, e.g., because the wind perturbed the system. Changes in the thermocline are then uniquely coupled to surface expressions through vertical dynamical modes. Knowledge of the forcing coefficients in the surface layer are assumed to completely control the deep ocean.

In a second experiment, labeled G2, the $\alpha$ are allowed to be nonzero in all four layers over the full data period, thus producing 8960 unknowns in 2590 equations (15). Here we make the extremely pessimistic assumption that disturbances leading to later changes in surface pressure can appear at depth within the model domain from unknown causes, e.g., missing model physics. It is plausible that such perturbations can enter the domain at depth across the lateral edges, but permitting them also within the interior represents an extreme case.

Two further experiments were performed which are a combination of the previous two scenarios. The first of

Table 2. Definitions of the Four Twin Experiments

\begin{tabular}{ll}
\hline Exp. & Comment \\
\hline G1 & $\alpha \neq 0$ only in surface layer for all $t$ \\
G2 & $\alpha \neq 0$ in all four layers for all $t$ \\
G3 & same as $G 1$, plus $\alpha \neq 0$ in all layers at $t=0$ \\
G4 & same as $\mathrm{G} 1$, plus $\alpha \neq 0$ in all layers at $t=0$ and \\
& along lateral boundaries for all $t$
\end{tabular}




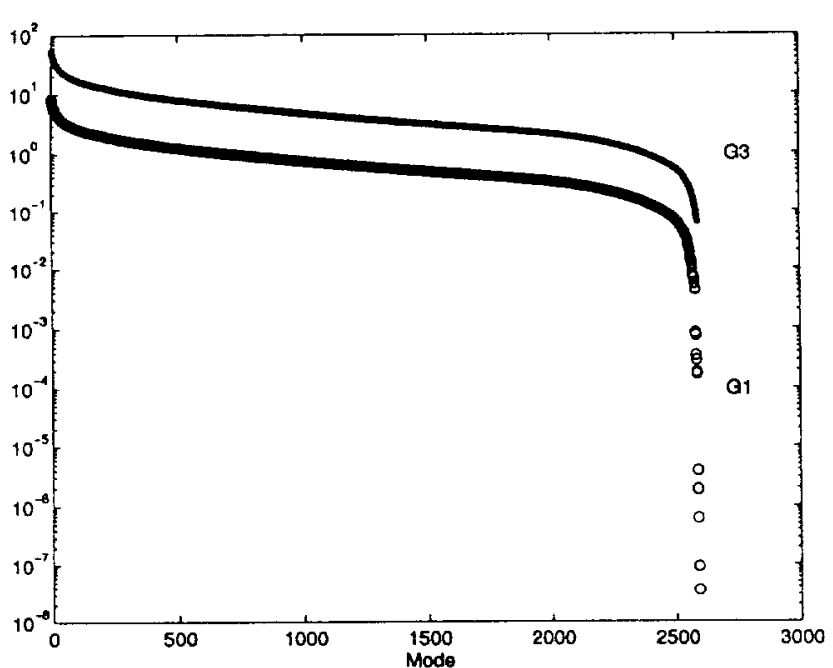

Figure 8. Singular value distribution from the two cases G1 and G3. See text and Table 2 for details on the experiments.

these, G3, is basically experiment G1, but with nonzero $\boldsymbol{\alpha}(0)$ in the full water column, equivalent to permitting an initial model correction over the full water column. Later developments are, however, through surface forcing perturbations alone. Finally, G4 includes in addition to G3, all $\alpha\left(t^{\prime}\right), t^{\prime}>0$, along the lateral boundaries of the coarse grid, thus accounting for perturbations which might enter into the estimation domain at depth from outside the domain.

The singular values $\lambda_{i}$ of the two cases G1 and G3 are depicted in Figure 8. Formally, both systems are full-rank (the same being true for the other two cases), with no singular values actually vanishing. For $\mathrm{G} 1$, the full rank means that the solution is completely resolved and would be perfect if the synthetic observations were actually perfect. There are five very small $\lambda_{i}$, however, and some rank deficiency is expected. Errors do arise from the loss of spatial resolution in moving to the coarse grid, and from edge effects in the averaging. When the five very small $\lambda_{i}$ and corresponding singular vector structures are dropped, some data and solution resolution is lost, basically confined to the tropics, and mainly at the beginning and end of the 1-year time period. A somewhat more realistic situation is obtained by taking the effective rank as $K=1500$, which implies a noise level of about $3 \%$ of the surface elevation variance. (Results are not very sensitive to the specific rank. The value of $K=1500$ was chosen here for consistency with the application to $\mathrm{T} / \mathrm{P}$ data described in the next section.)

For G3, and similarly for G2 and G4, the $\lambda_{i}$ vary only over about 2 orders of magnitude. Whether full rank or not, cases G2 to G4 are always formally underdetermined, and one normally expects to use prior statistical information and understanding in finding a best solution. In the spirit of exploring a somewhat pessimistic situation (G2), we assumed only that the solution $\hat{\alpha}$ has a variance inversely proportional to the layer thick-

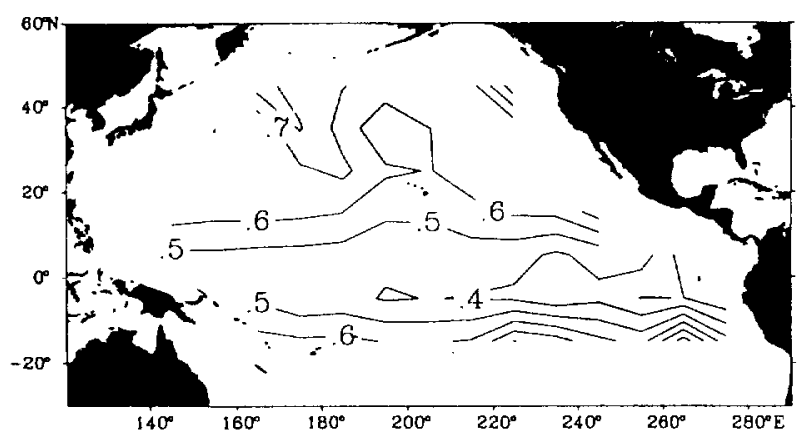

Figure 9a. Diagonal elements of the data resolution matrix $\mathbf{T}_{u}=\mathbf{U} \mathbf{U}^{T}$, which results from a rank reduction to $k=1500$ and plotted for $t=100$ days in geographical order.

nesses (smallest in the deep water). For G3 and G4, we altogether suppress any significant variances in $\hat{\alpha}\left(t^{\prime}\right)$ in the interior, except at $t^{\prime}=0$ for an initial correction (G3) and along the lateral edges in layer 2 and 3 for $t^{\prime}>0$ for G4. A useful refinement (not done here) would be to impose larger variances near western boundaries or near mean currents. The imposed variances could come from the model itself, or from observations of any kind.

G1 and G3 produce basically identical results, but the latter case is more physically attractive and we have chosen it as the standard "best case" to be described in more detail. Differences in the other experiments and the physical implications are discussed subsequently.

In the present artificial situation, the main issues are those of resolution. A typical mid-data-stream spatial pattern (corresponding to $t=100$ days) of data and solution resolution is displayed in Figure 9 on the coarse grid. The fields are obtained assuming an effective rank of $K=1500$, in which modes with small spatial scales and timescales are mostly suppressed. Generally, the data resolution (Figure $9 \mathrm{a}$ ) is enhanced in higher latitudes and is small along the equator, with minimum values residing along $5^{\circ} \mathrm{S}$ in the eastern basin. In addition, some fine structure is visible, which to a limited degree shows a correlation with the bathymetry, e.g., along the Emperor Seamount Chain and the Hawaiian

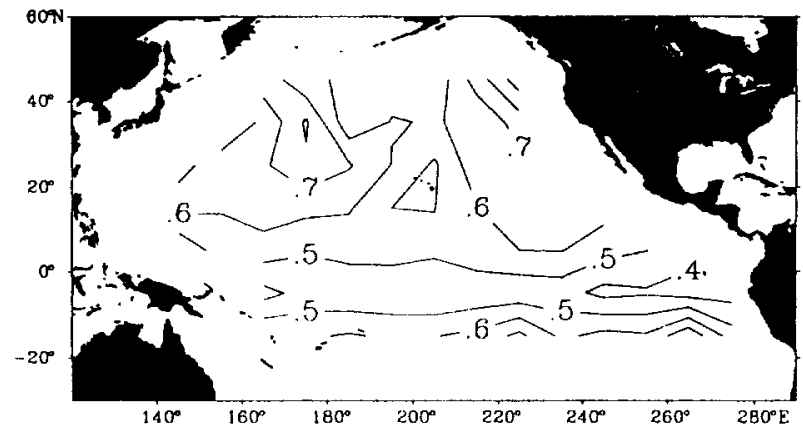

Figure 9b. Same as Figure 9a but for solution (parameter) resolution matrix $\mathbf{T}_{v}=\mathrm{VV}^{T}$ in the first model layer. 

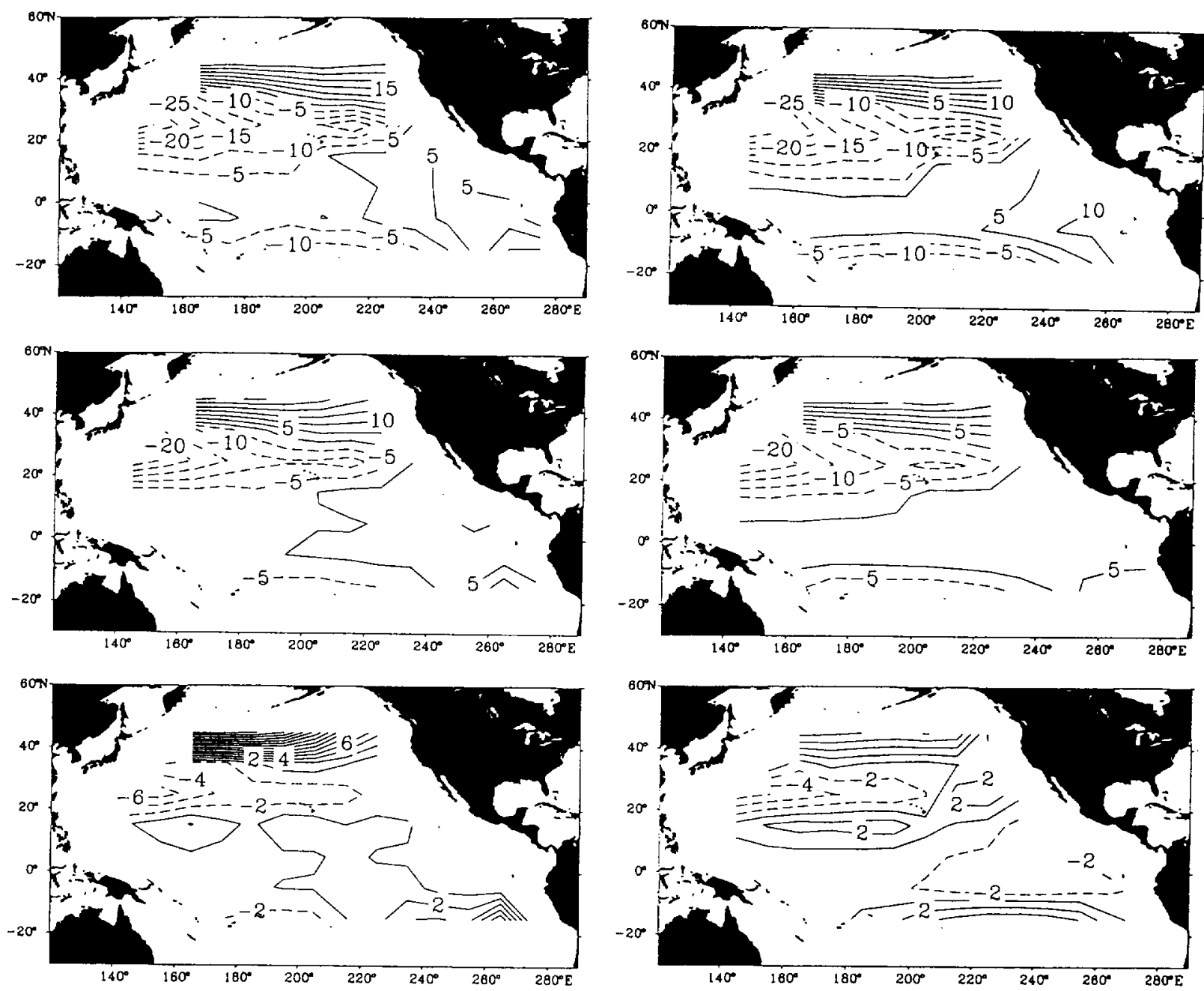

Figure 10. Time means of (left) $\hat{\delta p}(t)$ and (right) $\delta p_{o b s}(t)$ in centimeters in top three model layers (from top to bottom) at all 74 locations on the coarse grid.

Ridge. The fact that all values are significantly lower than unity indicates that no individual datum is fully resolved, i.e., the system lacks adequate information to distinguish individual equations from linear dependence on one another [Wunsch, 1996]. Owing to the presence of fast modes on large zonal scales, in the band $\pm 10^{\circ}$ around the equator, individual observations sampled at a 10 day interval are barely independent of each other. In particular, data from the eastern tropical Pacific are least important in constraining the solution. One physical reason is the presence of the East Pacific Rise, which hinders the penetration into the interior basin of the equatorial Rossby waves excited in that area. In addition, any Kelvin wave generated there soon disappears at its eastern border.

The solution (parameter) resolution for the same time step in the surface layer is shown in Figure 9b. Although slightly different in detail, it shows basically the same spatial pattern and amplitudes as appear for the data resolution. The similarity suggests a strong, but incomplete, dependence of the surface layer values of $\hat{\boldsymbol{\alpha}}$ on the local measurements. In general, $\hat{\boldsymbol{\alpha}}$ are determined as linear combinations of the elements of the true value $\alpha$, owing to a less than full rank system. Again the tropical area shows the largest deficiencies.

Based on the artificial model observations and the SVD of the Green's function matrix, corresponding coefficients $\hat{\alpha}$ were obtained from (19) with $K=1500$, and an estimate of the full model state was recovered subsequently from (21) to (22).

A comparison of the estimated pressure anomaly field $\delta \mathbf{p}$ with the "true" anomalies is shown in Figure 10 for the top three layers. Because the bottom layer is only partially covered by the coarse grid, it is not displayed here; results, however, are qualitatively very similar to what is found from layer 3 . Shown are instantaneous fields from $t=100$ days, which nonetheless represent typical situations.

In summary, "true" model fields are simulated in the top two layers, qualitatively and quantitatively. The rms misfits are reduced to about $10 \%$ of their original values in the top two layers (Figure 11a), with the misfit in layer 2 slightly exceeding that of the top layer. Corresponding cross-correlation coefficients are as high as 0.95 or above, right from the beginning and over most of the 1-year period. Although the cross correlation is 


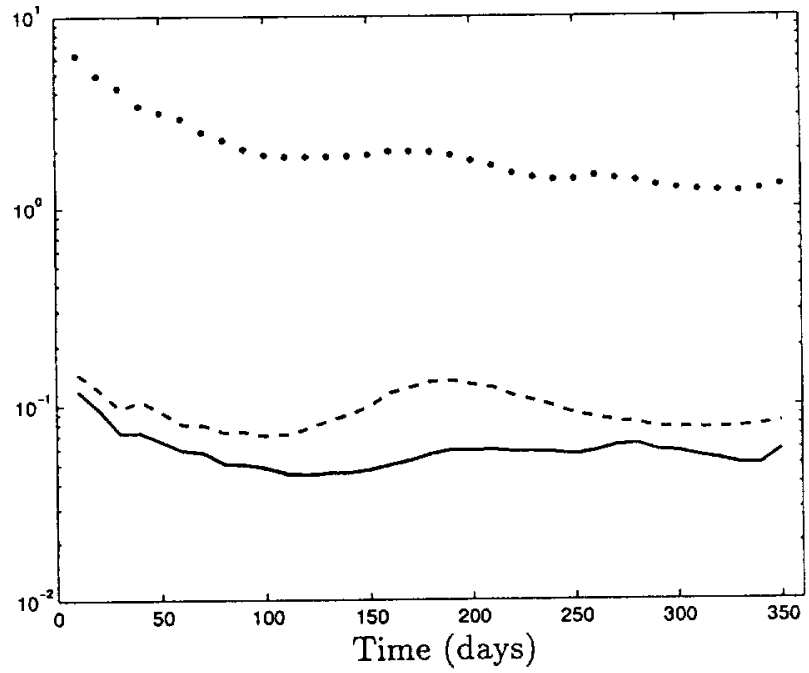

Figure 11a. The rms residuals from the twin experiment at $K=1500$ from top three model layers evaluated on all 74 grid points for each of the 35 time steps and normalized by the initial model/data misfit. Values from layers 1,2 , and 3 are drawn by solid, dashed, and dotted lines, respectively.

highly significant in the third layer, growing from an initial 0.5 to about 0.8 in the second half of the estimation period, related rms residuals vastly exceed the initial small discrepancy in layer 3.

A close inspection reveals the troublesome areas to be confined to the northern border of the coarse grid. There, the Green's functions, being predominantly barotropic in nature, do not project enough energy onto the first baroclinic mode, which is necessary to match the observations. The tendency is, however, in the right direction, with baroclinic modes increasingly gaining energy through the continuous reinitialization. Improved agreement from assimilation runs lasting for

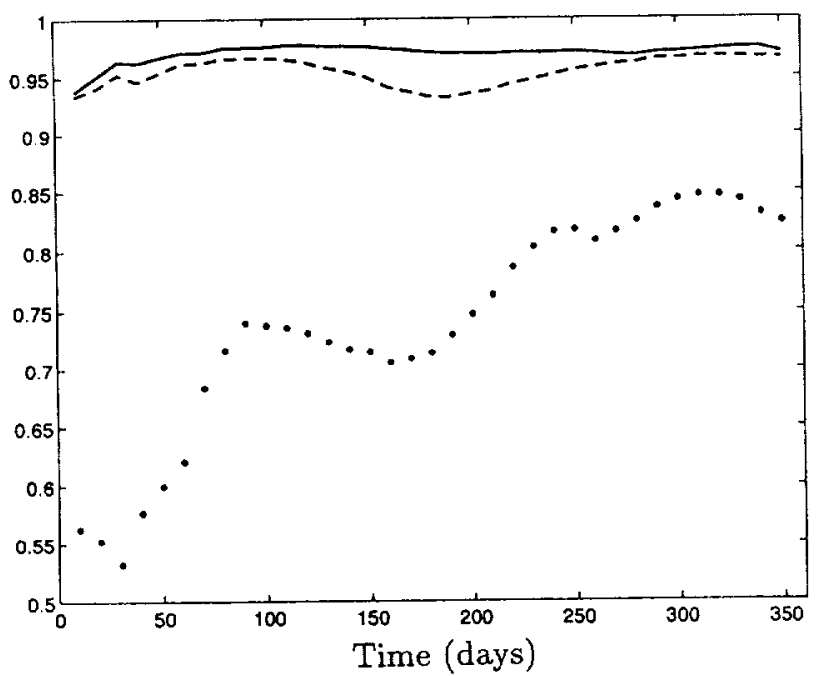

Figure 11b. Cross-correlation coefficient bedween $\hat{\delta p}(t)$ and $\delta p_{\text {ops }}(t)$ as a function of time. Values from layers 1,2 , and 3 are drawn by solid, dashed, and dotted lines, respectively.

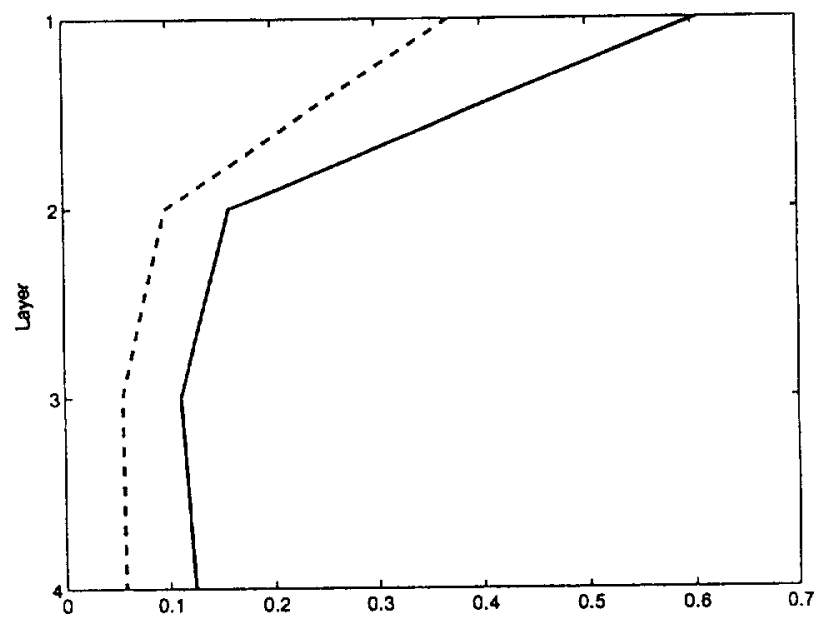

Figure 12. Space-time mean of the diagonal elements of the pressure resolution matrix given as $\mathbf{T}_{p}=$ $\mathbf{G}^{p} \mathbf{T}_{v}\left(\mathbf{G}^{p}\right)^{-1}$, averaged over the full coarse grid and time span of data for each layer at $K=1500$ (dashed) and $K=2590$ (full-rank, solid).

longer periods can be anticipated. Furthermore, the predominantly barotropic nature of the Green's function in these areas is an artifact of the low vertical resolution. Another area of potential problems can be found in the eastern tropical Pacific, where topographic effects are likely to be significant.

The reduced skill at depth is readily anticipated from a resolution analysis of the model and observations, and it is the availability of this information which would prevent any user of the method from falsely inferring that the results at depth are better than is warranted, when the true field was not actually known. Figure 12 depicts the pressure resolution $\mathbf{T}_{p}=\mathbf{G}^{p} \mathbf{T}_{v}\left(\mathbf{G}^{p}\right)^{-1}$ as a function of depth when averaged over the entire time span of data and over each layer for $K=1500$ and $K=2590$ (full rank). Here $\mathbf{T}_{v}$ is the solution resolution matrix as given in Figure 9. As in (21), the matrix $\mathbf{G}^{p}$ is complete, covering the whole space and time span, including all four layers, and $\left(G^{p}\right)^{-1}$ is the generalized inverse of this overdetermined system. Not surprisingly, the solution resolution degrades rapidly with depth, even for the full-rank case. Physically, the result means that pressure anomalies at great depths have unobservable consequences at the sea surface over time spans of 1 year. Although longer records will improve these results, as deep anomalies generate observable surface changes over time, it was the anticipation of this situation that led Munk and Wunsch [1982] to propose the complementarity of altimetry and ocean acoustic tomography, the latter providing the resolution at depth, which is difficult for altimetry.

Temperature anomalies $\hat{\delta \theta}$ estimated from (22) are compared with the "true" fields in Figure 13, again taken from $t=100$ days and representing typical instantaneous situations. The poorest visual agreement between the inferred and true temperature fields is found in the surface layer, where the true model state is relaxed continuously toward Levitus's climatology. The 

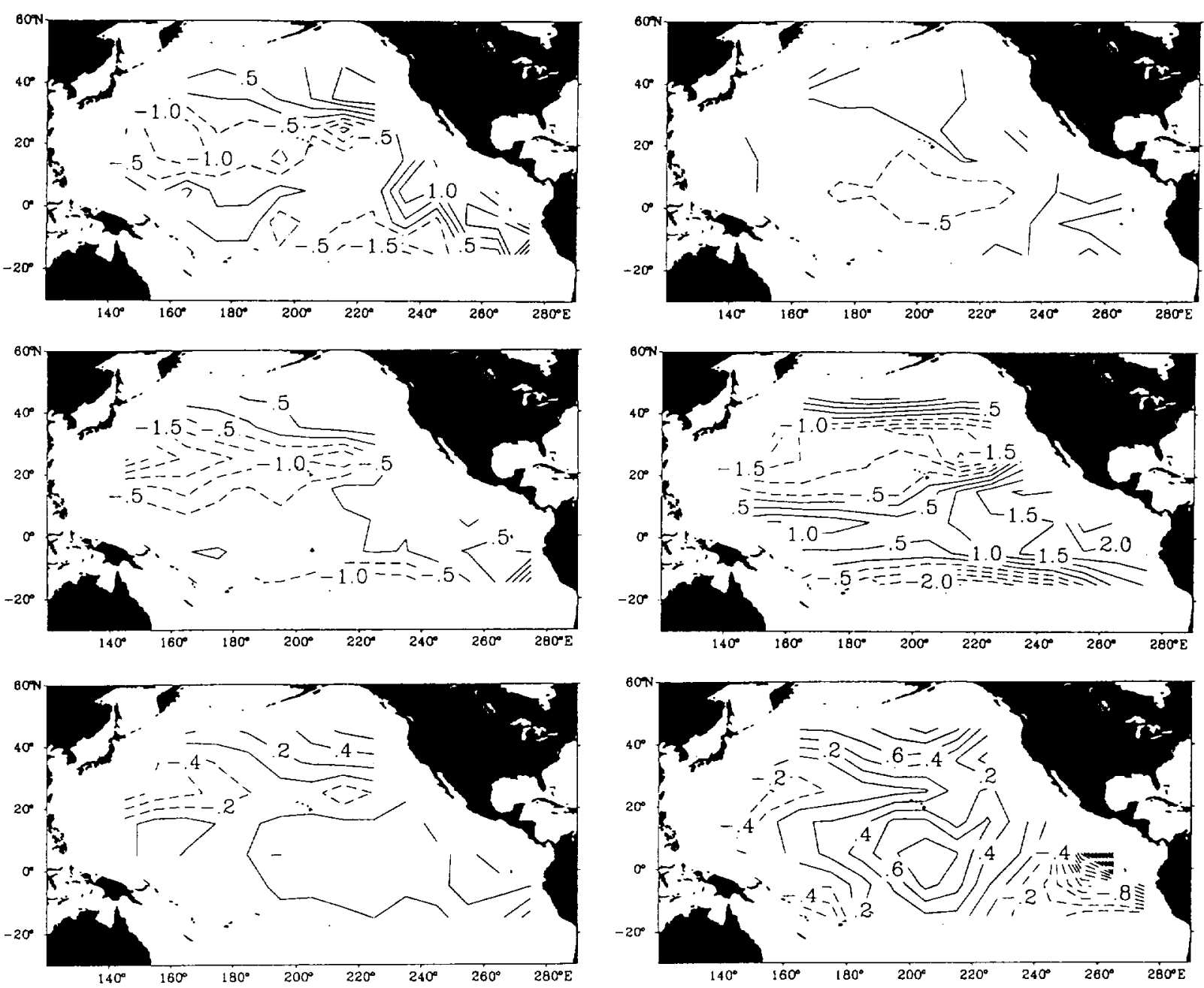

Figure 13. Time mean (left) $\delta \hat{T}(t)$ and (right) $\delta T_{\text {obs }}(t)$ in degrees Celsius in top three model layers (from top to bottom) at all 74 locations on the coarse grid.

rms difference is about $1^{\circ} \mathrm{C}$ (Figure $14 \mathrm{a}$ ), and the cross correlation is as low as 0.4 , yet significant, over the entire period (Figure 14b). We infer that the poor agreement indicates the inconsistency of the Levitus numbers with the model physics in the surface layer. In contrast, general agreement of the results with the "truth" is found in the second layer, where the mean correlation is 0.6. As in the top layer, the largest residuals are confined to the tropical ocean, where the induced uncertainties are largest. As a result, the temperature is systematically underestimated by $2^{\circ}$ to $3^{\circ} \mathrm{C}$ (rms misfit is again close to $1^{\circ} \mathrm{C}$, globally). In the third layer, the correlation between observations and estimations is only marginally significant. Again an estimate with increased accuracy in the deep ocean can be anticipated from longer data sets because the dynamical adjustment timescale in the deep ocean is 1 to 2 orders of magnitude larger than near the surface.

Summarizing the results from the various case studies, no significant difference in the estimated state measured in rms residual amplitudes of the pressure and temperature fields was obtained from $\mathrm{G} 1$ compared to G3. The effect of permitting $\hat{\alpha}(0) \neq 0$ in all layers leads only to slightly decreased residuals in the top two layers during the first 50 days, but a small degradation below. Permitting subsurface perturbations over the full time span during $G 2$ reduces the residuals in surface pressure somewhat, consistent with the increased degrees of freedom. In the second layer and below, which are not constrained during the inversion, however, a significant degradation of the estimated state relative to the truth was found as compared to the purely surface driven case. Including only coefficients along lateral boundaries (G4) did not show any benefit near the surface (as compared to G1 and G3), but led to some degradation in layers 3 and 4 .

\section{TOPEX/POSEIDON Altimetry}

Our central goal is the estimation of the present state of the ocean given real altimeter data, and in the following, we will discuss the results obtained with $T / P$ data from the first year of the mission. $T / P$ data from the 1-year period December 21, 1992, to December 3,1993 , corresponding to repeat cycle 10 through 44, were edited and corrected as described by Stammer and Wunsch [1994] and King et al. [1994]. Only two 


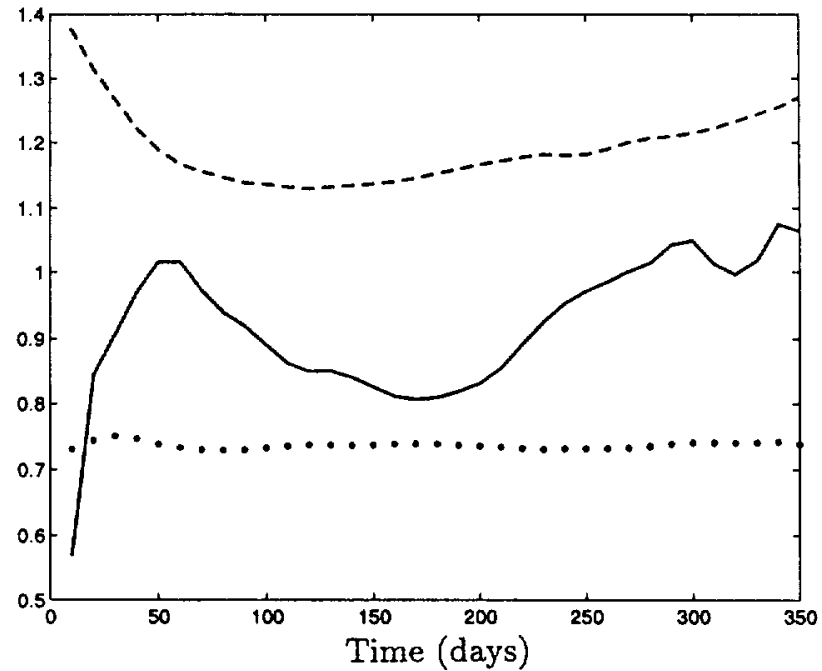

Figure 14a. The rms temperature residuals from the twin experiment at $K=1500$ from top three model layers evaluated on all 74 grid points for each of the 35 time steps. Values from layers 1,2 , and 3 are drawn by solid, dashed, and dotted lines, respectively.

significant modifications of the standard merged $T / P$ geophysical data records produced by the project $[\mathrm{Be}$ nada, 1994] should be noted here because they influence the results and the underlying errors. First, to obtain observations of the dynamic sea surface height (SSH), we referenced the $\mathrm{T} / \mathrm{P}$ observations to a hybrid geoid (JGM-2/OSU91a) derived by Nerem et al. [1994], and Rapp et al. [1991], where the former is used to spherical harmonic degree 70 and the latter beyond that to degree 360 . Second, the tidal correction provided by the $T / P$ project was replaced by those estimated by $\mathrm{Ma}$ et al. [1994] from the first year of T/P data (version UT/CSR 1.4). The resulting mean field of this 1 -year period is

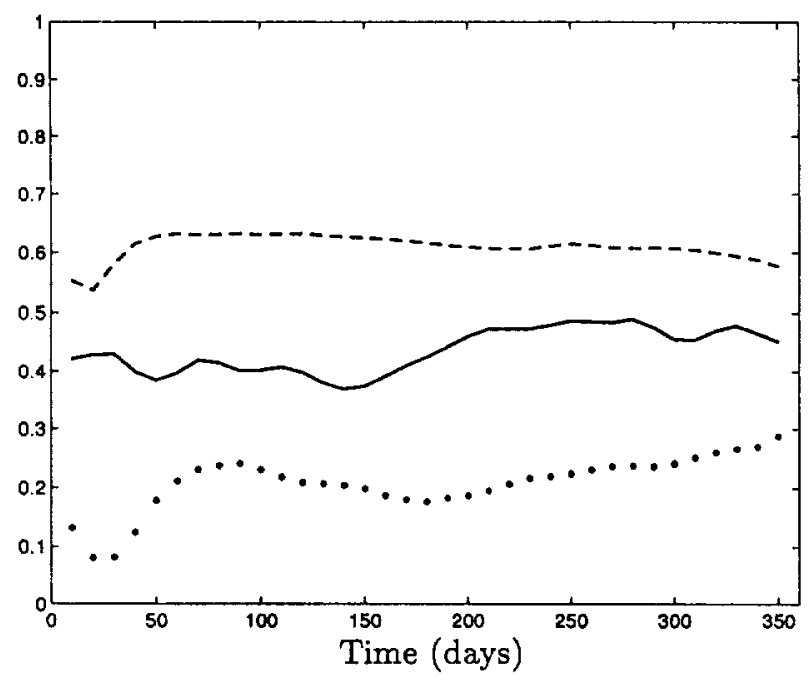

Figure 14b. Cross-correlation coefficient between $\hat{\delta \theta}(t)$ and $\delta \theta_{\text {obs }}(t)$ as a function of time. Values from layers 1,2 , and 3 are drawn by solid, dashed, and dotted lines, respectively.

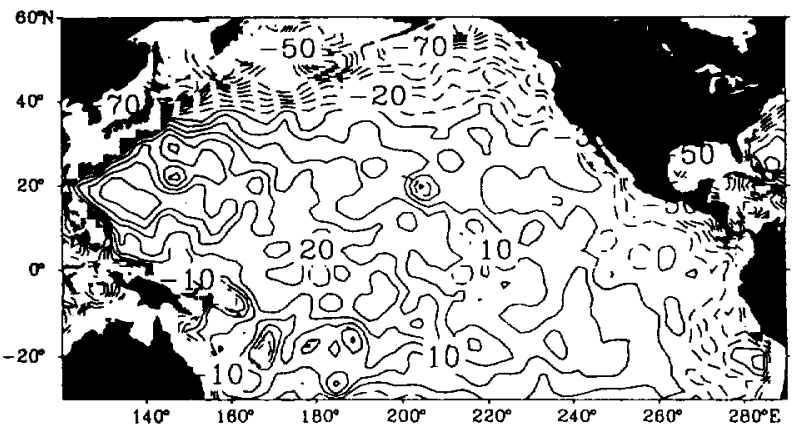

Figure 15a. Mean TOPEX/POSEIDON sea surface height observation inferred from repeat cycle 10 to 44 relative to the JGM-2 geoid. The contour increment is $10 \mathrm{~cm}$.

shown in Figure 15a. A mean difference between the model-predicted surface elevation and that observed by $T / P$ is shown in Figure 15b. Apart from model errors, the dominant errors in Figure 15b should be those of the geoid. Although geoid errors are geographically variable and strongly correlated, for present purposes they will be treated as homogeneous and "white."

To form a vector of observations, for each individual 10-day period, instantaneous fields similar to Figure $15 \mathrm{~b}$ were averaged in $10^{\circ} \times 10^{\circ}$ areas which coincide with the coarse grid. Resulting fields were subsampled along the grid points given in Figure 1d. The model climatology and Green's functions were used with the T/P data in the same way as employed for the twin experiments. It should be emphasized that the resulting model/data differences are based on the absolute T/P SSH, not just the time-dependent part.

We confine the discussion to G3, which was identified in the previous section as being the physically most plausible situation. The ability to make inferences about the ocean circulation is directly dependent upon the noise level in the observations. The discussion of the noise level is slightly subtle here because we are simultaneously discussing both the time mean circulation, which is sensitive to geoid errors, and the low-frequency variability (dominated by the annual cycle), which is to first order, independent of such errors. A number of studies of geoid error [Nerem et al., 1994; Tsaoussi and Koblinsky, 1994] would lead to a rank $K=500$ corre-

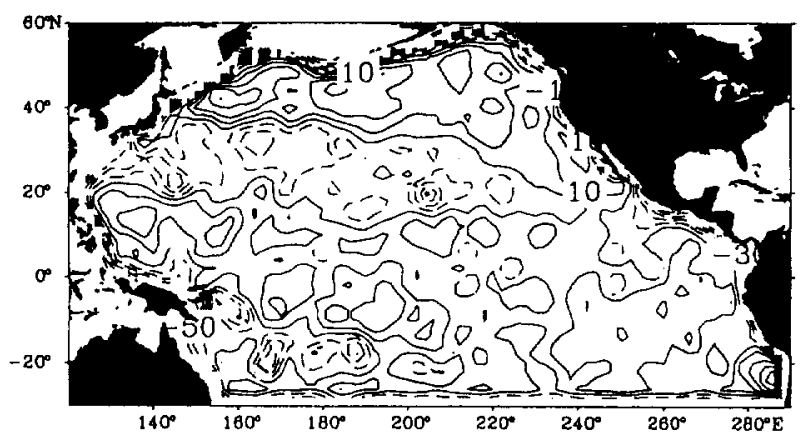

Figure 15b. The difference of this mean sea surface height field relative to the reference model state. The contour increment is $10 \mathrm{~cm}$. 

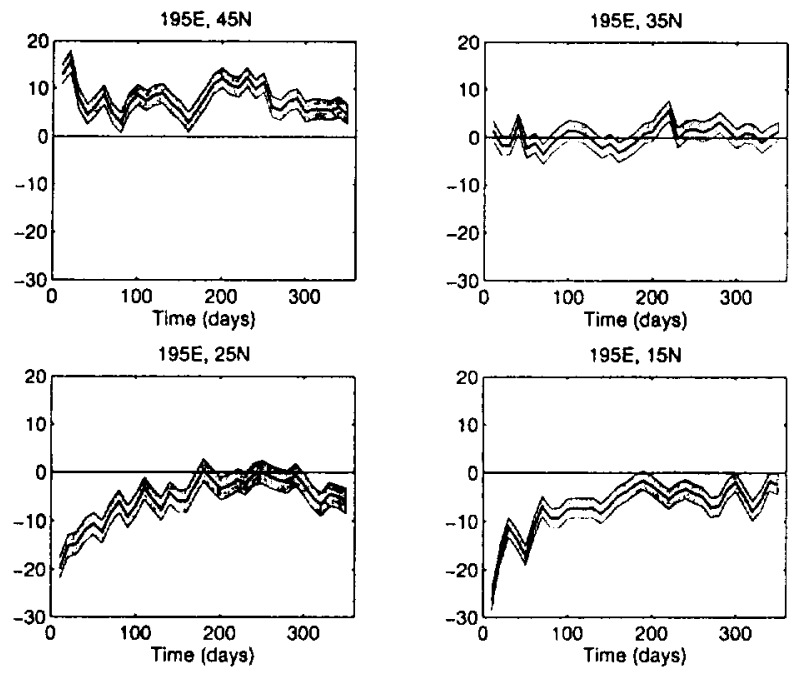

Figure 16. Forcing coefficients $\alpha(t)$ in centimeters of equivalent surface elevation at various representative locations. Shaded areas correspond to the uncertainty of the coefficients as given by $\left(\operatorname{diag}\left\{\mathbf{P}_{\alpha \alpha}\right\}\right)^{1 / 2}=$ $\left(\sigma^{2} \operatorname{diag}\left\{\mathbf{V}_{K} \Lambda_{K}^{-2} \mathbf{V}_{K}^{T}\right\}\right)^{1 / 2}$.

sponding to an error at about $20 \%$ of the total variance. This rank will be used for studying the time mean. In examining the annual cycle, however, the rank is taken to be $K=1500$, corresponding to a noise level $\sigma$ of about $2.5 \mathrm{~cm}$ (roughly a $5 \%$ error level), which is more appropriate to the time-dependent measurements, but the time means are removed from these results, as they are regarded as noise-dominated. An alternative approach would be to completely separate the discussion of time-mean and time-dependent fields by generating a new data set from the time derivatives of the observations. The present method is simpler, however.

In this preliminary attempt, no use was made of known correlations in the error fields (resulting, generally in a reduction in the skill of the data compared to what is actually possible). Use of such correlations is a high priority for future work.

Consider the higher rank system, $K=1500$. The time histories of $\hat{\boldsymbol{\alpha}}_{j}\left(t^{\prime}\right)$ and their uncertainties, $\pm\left(\sigma^{2} \operatorname{diag}\left\{\mathbf{P}_{\alpha \alpha}\right\}\right)^{1 / 2}$, are shown in Figure 16 at a few representative locations $j$. Uncertainties are computed

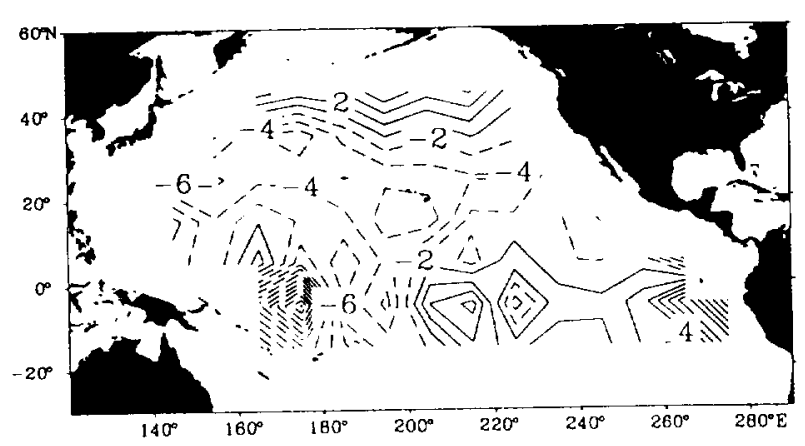

Figure 17. Time mean coefficients $\overline{\hat{\alpha}}$ as a function of geographical positions. by taking into account only the first term on the righthand side of (20), omitting the unknown contributions from the null space. In general, the largest amplitudes occur, as expected, where initial model/data differences are large at $t^{\prime}=0$, representing the initialization errors with amplitudes gradually decreasing subsequently toward an asymptotic level.

Systematic differences between the model and the data appear as corrections of uniform sign over large areas and long times. Figure 17 shows the average $\hat{\alpha}$ over a full year at rank 1500 . As in section 3 , we can interpret these mean coefficients as representing the consequences of a systematic error in the forcing, e.g., a wrong wind stress boundary condition, or incomplete model physics. The correction keeps the model tracking the observations by depressing the subtropical gyre and uplifting it at higher and lower latitudes in a manner one anticipates from the mean model/data difference (Figure 15b).

Time histories of the surface pressure corrections $\hat{\delta \mathbf{p}_{i}}(t)$ are shown in Figure 18 for a few representative locations $i$, along with the uncertainty estimates $\left(\operatorname{diag}\left\{\mathbf{P}_{p p}\right\}\right)^{1 / 2}$ of $\hat{\delta p}$ from (23). As required, the estimates follow the observations in a slightly smoothed manner and generally within the uncertainty limits.

In summary, the method produces reasonable results, both visually and in terms of the prior statistical estimates. As always, however, the degree of agreement of model and data is sensitive to the choice of rank, i.e., the assumed noise level of model and data. Observations are reproduced identically in the surface pressure at full rank. Assuming an error of $2.5 \mathrm{~cm}(K=1500)$, the observed variance and its seasonal variation are simulated (Figure 19a), with major deviations limited to the initial 1-month period. Accounting for geoid uncertainties $(K=500)$, though, leads to a variance level slightly lower than the observed mean, and the estimates do not show a seasonal cycle.
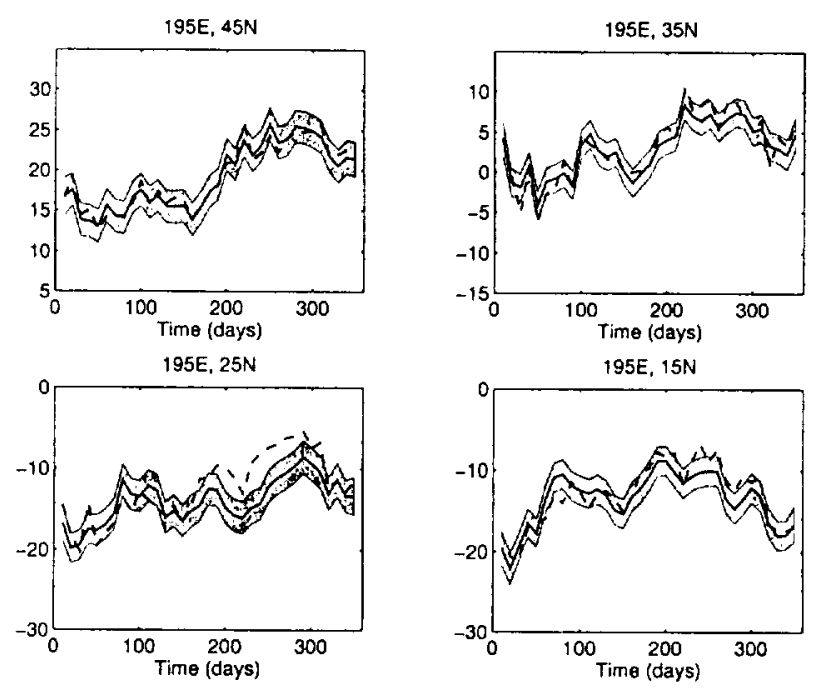

Figure 18. Estimates $\hat{\delta p}(t)$ (solid line) and observations $\delta p_{\text {obs }}(t)$ (dashed line) at the same representative positions as in Figure 16. Shading represents the uncertainties of $\hat{\delta p}(t)$, obtained from $\mathbf{P}_{p p}=\mathbf{G}^{p} \mathbf{P}_{\alpha \alpha} \mathbf{G}^{p T}$. 


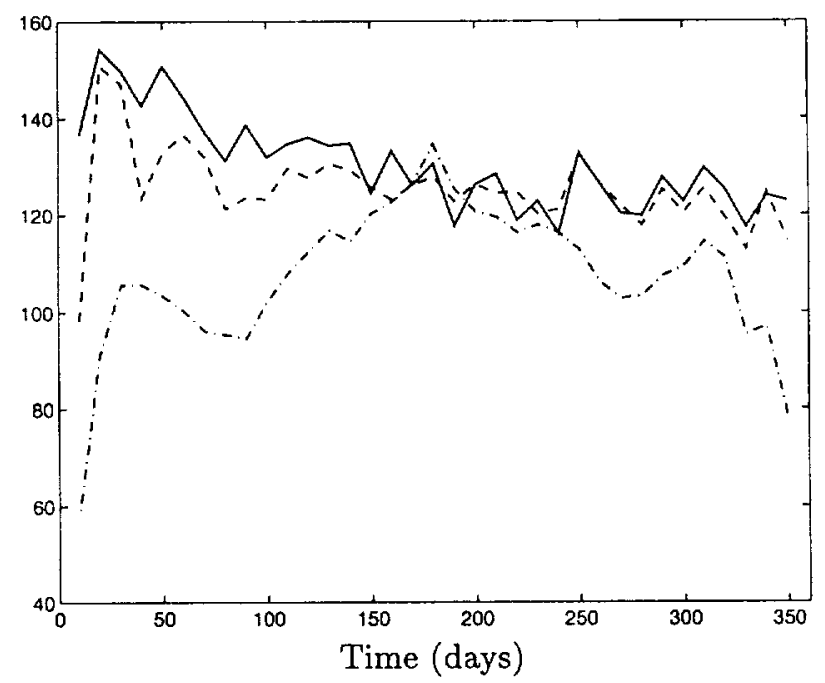

Figure 19a. Variance as it is observed (solid line) and simulated at rank $K=1500$ (dashed line) and $K=500$ (dash-dotted line).

The asymptotic rms residuals at $K=500$ indicate that prior error assumptions are consistent with the results, leading to a reduction in residuals close to $20 \%$ of the observed variance (Figure $19 \mathrm{~b}$ ). For $K=1500$ the error reduction is actually larger than anticipated, leading to an rms noise level of $3 \%$ (instead of the assumed $5 \%)$.

\subsection{Time Mean Corrections}

Here we focus on the time mean circulation from the rank 500 estimate. Figure 20 shows the absolute sea surface elevation field in the OGCM before and after correction by addition of the linear model estimates. The observations and inversion have forced a weakened subtropical gyre in the OGCM with its center shifted southward by about $10^{\circ}$ and with its zonal extent confined to the western part of the basin, approximately

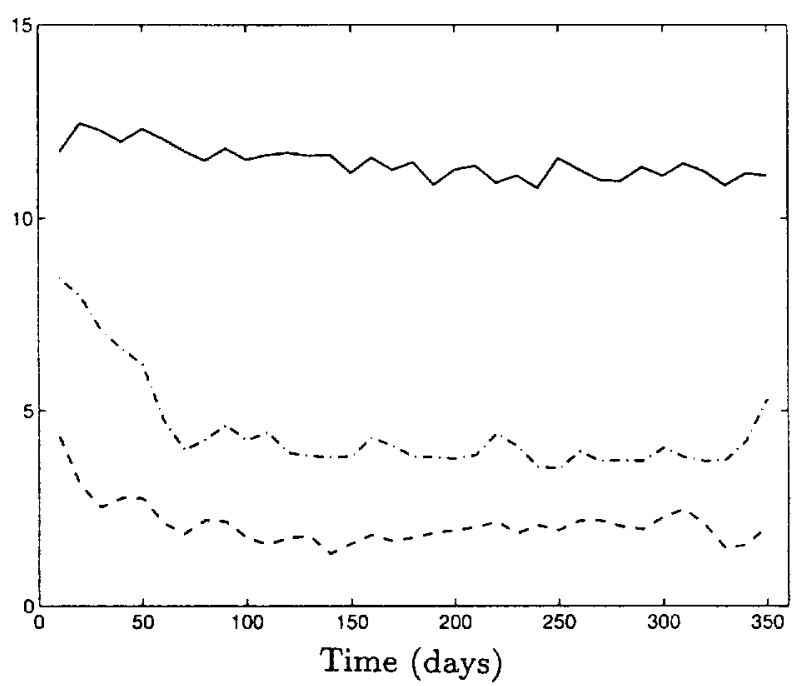

Figure 19b. The rms residuals obtained at $K=1500$ (dashed line) and $K=500$ (dash-dotted line), as compared to initial model/data misfit (solid line).
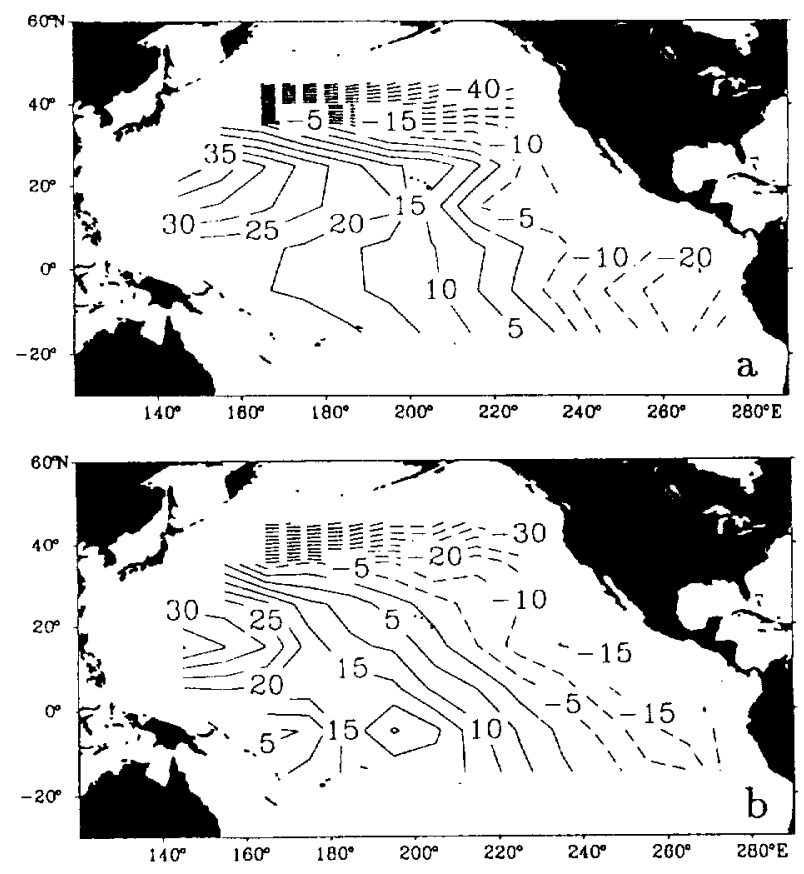

Figure 20. Absolute model sea surface elevation field (a) before and (b) after correction by the data. The contour increment is $5 \mathrm{~cm}$.

west of the Hawaiian Ridge. As compared to the T/P observations given in Figure 15a, many of the spurious small-scale anomalies, which appear to be associated with geoid uncertainties, have been reduced by forcing dynamical consistency on the motions.

The vertical structure of the absolute pressure in the basic state is shown in the left panel of Figure 21 along with the associated geostrophic flow. The time-mean correction in pressure to the model first estimate and the associated flow are shown the right panel of the figure. In general terms, the large-scale gyre is reduced from the original OGCM estimate by about $1 \mathrm{~cm} / \mathrm{s}$ to $2 \mathrm{~cm} / \mathrm{s}$ in the top two layers. In contrast, the cyclonic deep circulation is increased below $1000 \mathrm{~m}$ depth. The northern part of the correction has a simple, barotropiclike vertical structure, but has a more complex structure in low latitudes, associated with the eastward flowing equatorial counter current and with weak westward flow beneath of it.

The current and pressure anomalies are associated with temperature (density) anomalies at all depths. Some impression of the corrections required to these fields in the OGCM may be seen in Figure 22 along two meridional sections at $165^{\circ} \mathrm{E}$ and $215^{\circ} \mathrm{E}$. Consistent with the large-scale changes in sea level, the underlying thermocline is cooled or warmed in such a manner that the vertically integrated steric changes in density follow the required changes in sea level.

\subsection{The Seasonal Cycle}

Because of the heavy spatial filtering, the timevarying signal remaining in the $T / P$ data is dominated by seasonal changes in SSH with some minor amplitude fluctuations on timescales of 50 days superimposed 

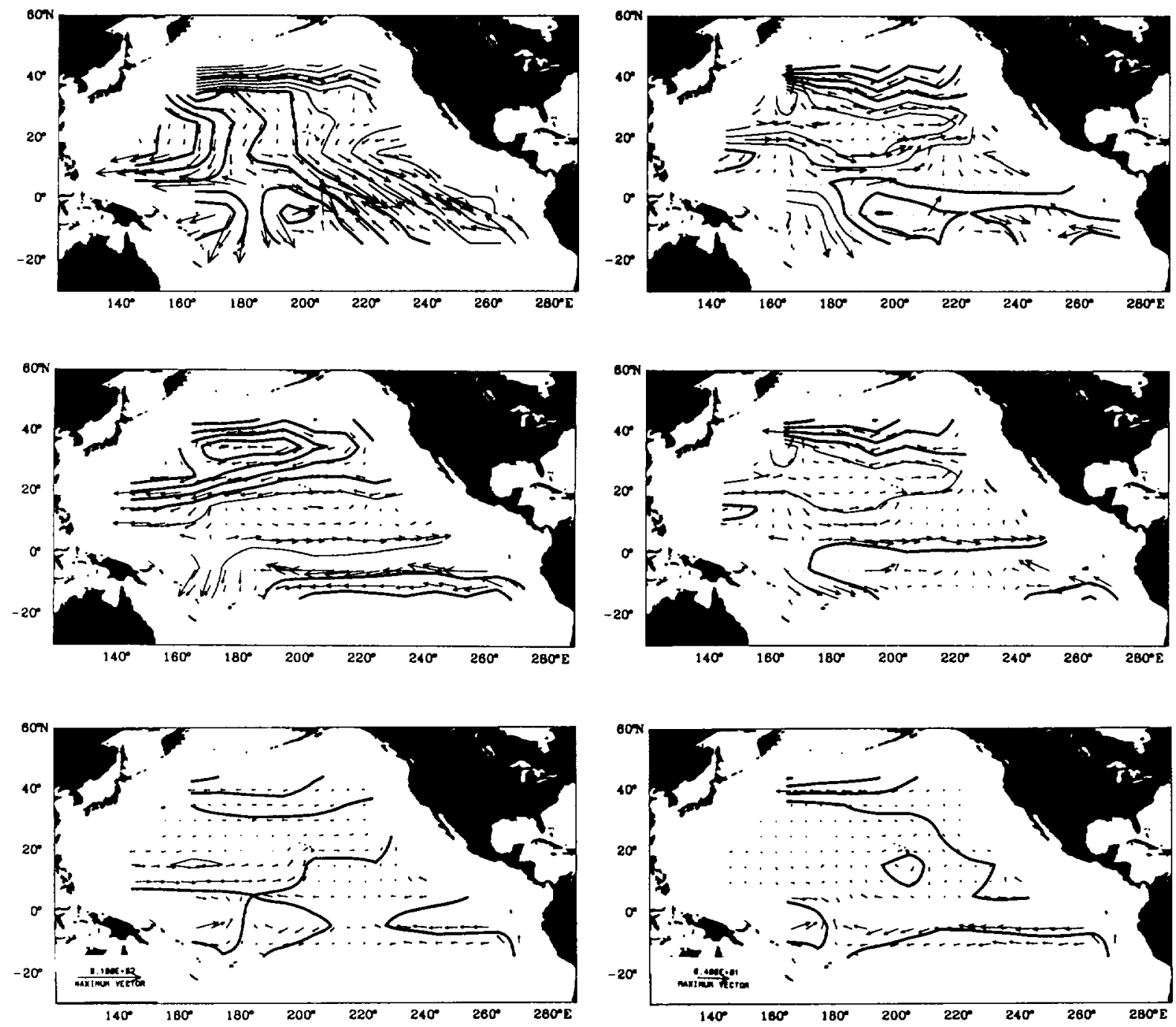

Figure 21. (left) Absolute pressure in the basic state along with the associated geostrophic flow for the top three model layers (from top to bottom). A spatial mean pressure has been subtracted from each field, and positive and negative values are drawn by bold, and thin lines, respectively. (right) Time mean corrections in absolute pressure and related geostrophic flow which are associated with the estimate given in the left panel. The contour increment is $5 \mathrm{~cm}$. The reference vectors represent $10 \mathrm{~cm} / \mathrm{s}$ (left) and $4 \mathrm{~cm} / \mathrm{s}$ (right).

(Figure 18). We therefore focus here on the seasonal signal. Figure 23 shows estimates of the surface elevation anomalies relative to the 1 -year mean following the inversion for the $\hat{\alpha}$ at $K=1500$. The fields are averaged over the four seasons of the year and are essentially the same as in Plate 3 of Stammer and Wunsch [1994]. In contrast to their plate, however, the present one is consistent both with the data and with the model dynamics. Also shown are the current field anomalies associated with the annual cycle elevation corrections. Most of these flows are very weak, in agreement with the theoretical estimates of Gill and Niiler [1973]. The exception is in the tropics, where the seasonally reversing North Equatorial Current and the Equatorial Counter Current of the order of $2 \mathrm{~cm} / \mathrm{s}$ are visible. (These numbers will increase when the model resolution is improved.)
The vertical structure of the seasonal current anomalies can be seen in Figure 24 in a section of the zonal velocity component along $185^{\circ} \mathrm{E}$. The most interesting feature is the secondary maximum occurring near $30^{\circ} \mathrm{N}$ at about $1000 \mathrm{~m}$. This structure, which is located in the vicinity of the Hawaiian Ridge becomes a prediction which could be tested against in situ observations. Otherwise, the corrected OGCM shows a large-scale current response with vertically coherent structures over the bulk of the section, with the exception of the tropical current fluctuations, which tend to be surface intensified.

The seasonal current and pressure anomalies are necessarily associated with temperature (density) changes at all depths, some of which are shown in Figure 25 along the same meridional section at $185^{\circ} \mathrm{E}$. Such results are equivalent to an estimate of the change in heat 

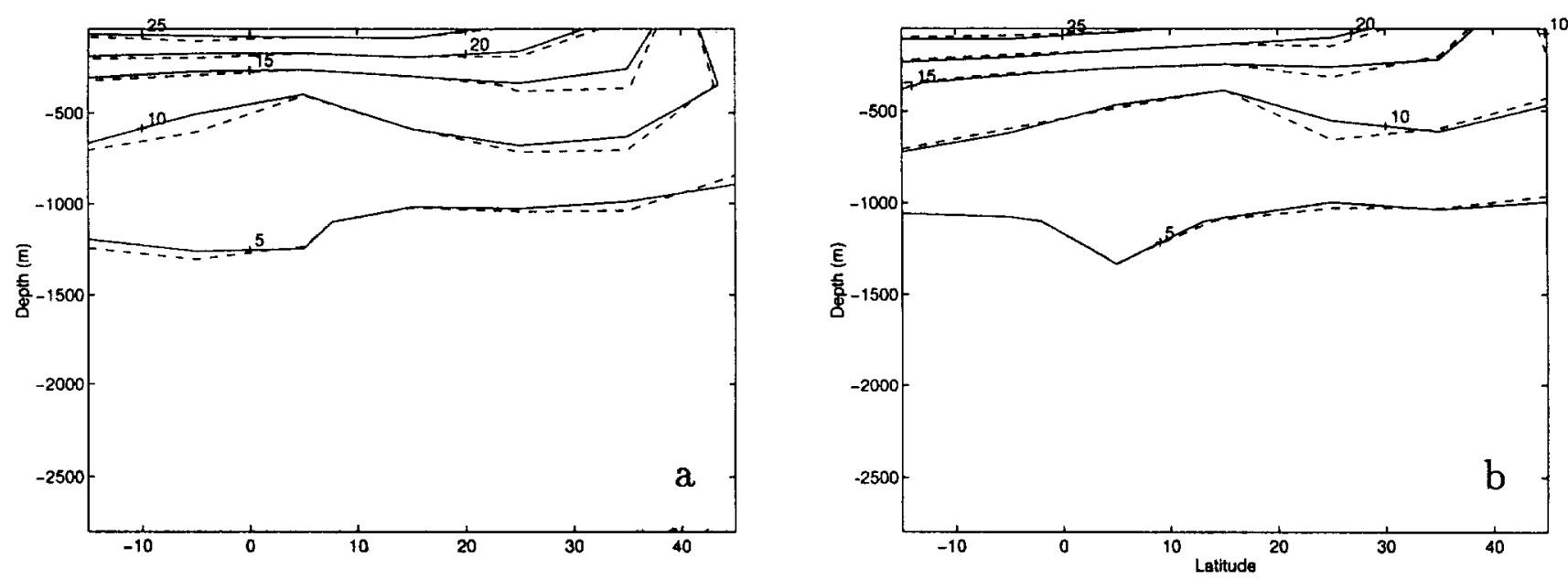

Figure 22. Meridional sections of temperature along (a) $165^{\circ} \mathrm{E}$ and (b) $215^{\circ} \mathrm{E}$ showing the temperature field before (dashed line) and after correction (solid line) by the $\mathrm{T} / \mathrm{P}$ data. Contour increment is $5^{\circ} \mathrm{C}$.

content of the ocean through the seasons. Temperature changes of close to $1^{\circ} \mathrm{C}$ are found in the surface layer. Those values are fairly small and should not be confused with observed surface temperature fluctuations, since they represent vertical integrals over the top 100 m. A simple calculation leads to an associated steric height change in sea level

$$
\delta \zeta_{s}=-\rho_{0}^{-1} \int_{-100}^{0} \alpha \delta \theta d z
$$

by $\delta \zeta_{s}=2 \mathrm{~cm}$, consistent with the estimated am-
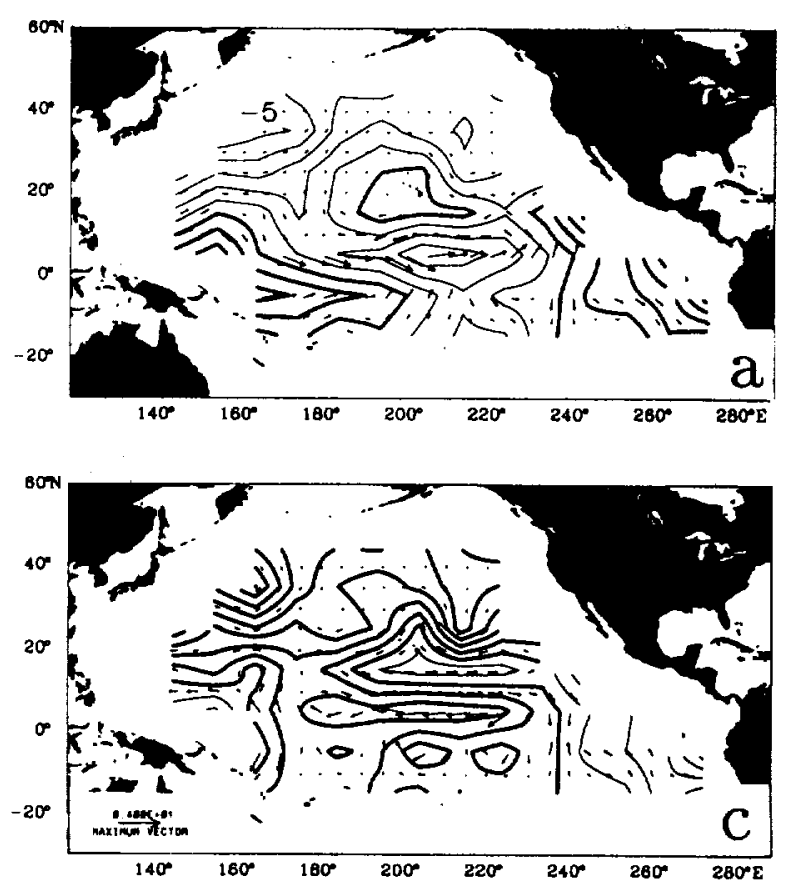

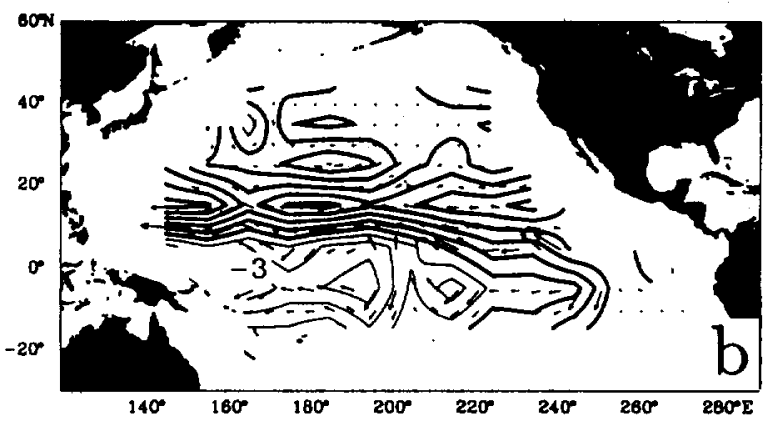

plitudes. On the other hand, seasonal temperature changes of the order of $0.1^{\circ} \mathrm{K}$ at $1000 \mathrm{~m}$ depth and below are artificially large. The relatively low vertical OGCM resolution enhances the vertical extent of responses to surface forcing and exaggerates temperature fluctuations in the main thermocline. (Recall the vertical resolution discussion in the twin experiments.)

We claim, then, that the model/data combination produces a better estimate of the seasonal cycle than either can do alone. The reader may, however, object that models such as the GFDL OGCM used here, are notoriously unable to properly compute the annual cy-

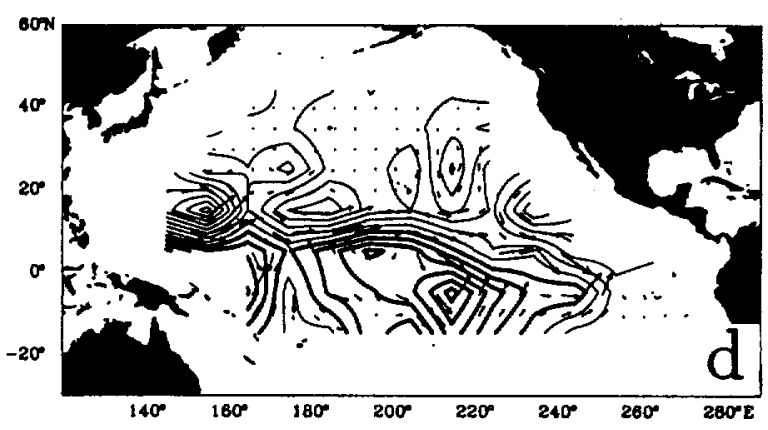

Figure 23. Estimates of seasonal surface elevation anomalies relative to the 1-year mean and related geostrophic currents. Fields represent (a) spring, (b), summer, (c) fall, and (d) winter, with spring starting at the beginning of March. Positive and negative values are drawn by bold, and thin lines, respectively. Contour increment is $1 \mathrm{~cm}$. The reference vector represent $4 \mathrm{~cm} / \mathrm{s}$. 

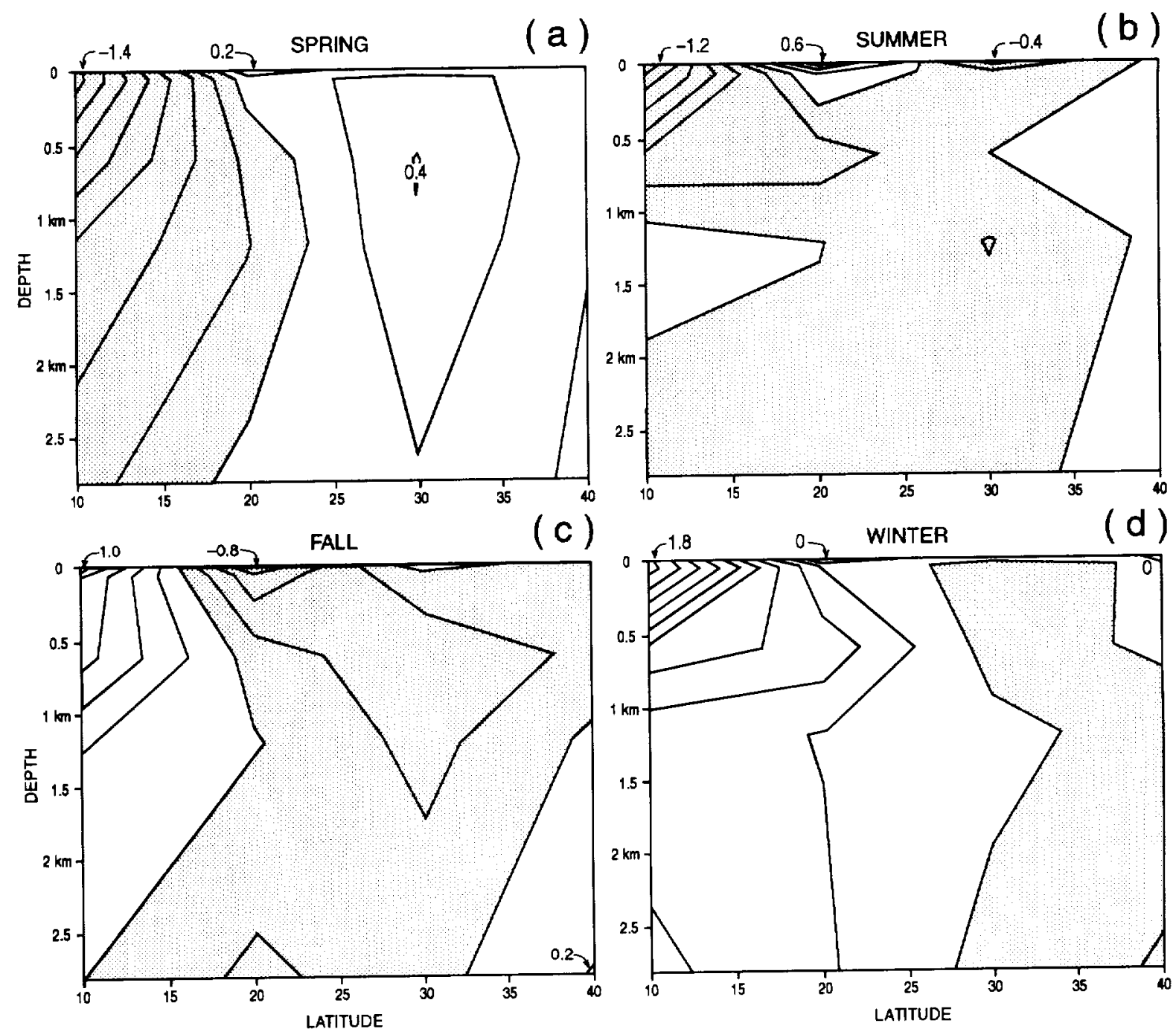

Figure 24. Meridional section of estimated zonal component of seasonal current anomalies along $185^{\circ} \mathrm{E}$. Fields represent (a) spring, (b), summer, (c) fall, and (d) winter. Contour increment is $0.2 \mathrm{~cm} / \mathrm{s}$ in all panels, and negative values are shaded.

cle of heating and cooling [Sarmiento, 1986, Stammer et al., 1996]. They generally fail to import sufficient heat into the oceanic interior across the sea surface during the heating season, thus producing a steric contribution to sea level which is too small. In the present circumstances, the observed sea level change is being forced onto the OGCM by the Green's functions. However, to the extent that a pressure-excited Green's function mimics the response from thermal driving once the thermal anomaly has been injected to depth, the results here should nonetheless be quite accurate.

An issue will arise, however, in a model with much higher near-surface resolution. Direct thermal injection would be expected to penetrate only to about $300 \mathrm{~m}$, whereas a mechanically driven perturbation, such as a wind-field anomaly would likely (but not necessarily) penetrate much deeper. In such a model, Green's function responses for thermal forcing must be computed for perturbations with a much shallower vertical penetration than from mechanical forcing. In the present case, the very thick top layer precludes distinguishing the two vertical scales, but we believe the results shown are as accurate a depiction of the thermal response as can be achieved with such low resolution.

\subsection{Residuals}

The estimation method has proceeded on the assumption that errors in the linear model and data have a white noise character. In general this is not correct, and residuals, although consistent with the general error budget, show strong geographically correlated structures, and which at various locations greatly exceed the assumed error level. In Figure 26 the time-averaged residuals $\overline{\hat{\mathbf{n}}}=\overline{\mathbf{y}_{\text {obs }}-\hat{\mathbf{y}}}$ at rank $K=500$ are shown. Values of up to $10 \mathrm{~cm}$ in amplitude can be found in various locations, but predominantly in the tropics. These residuals are static elements of the data which are not consistent with the underlying error assumptions and linear model physics.

Such geographically correlated components in the 

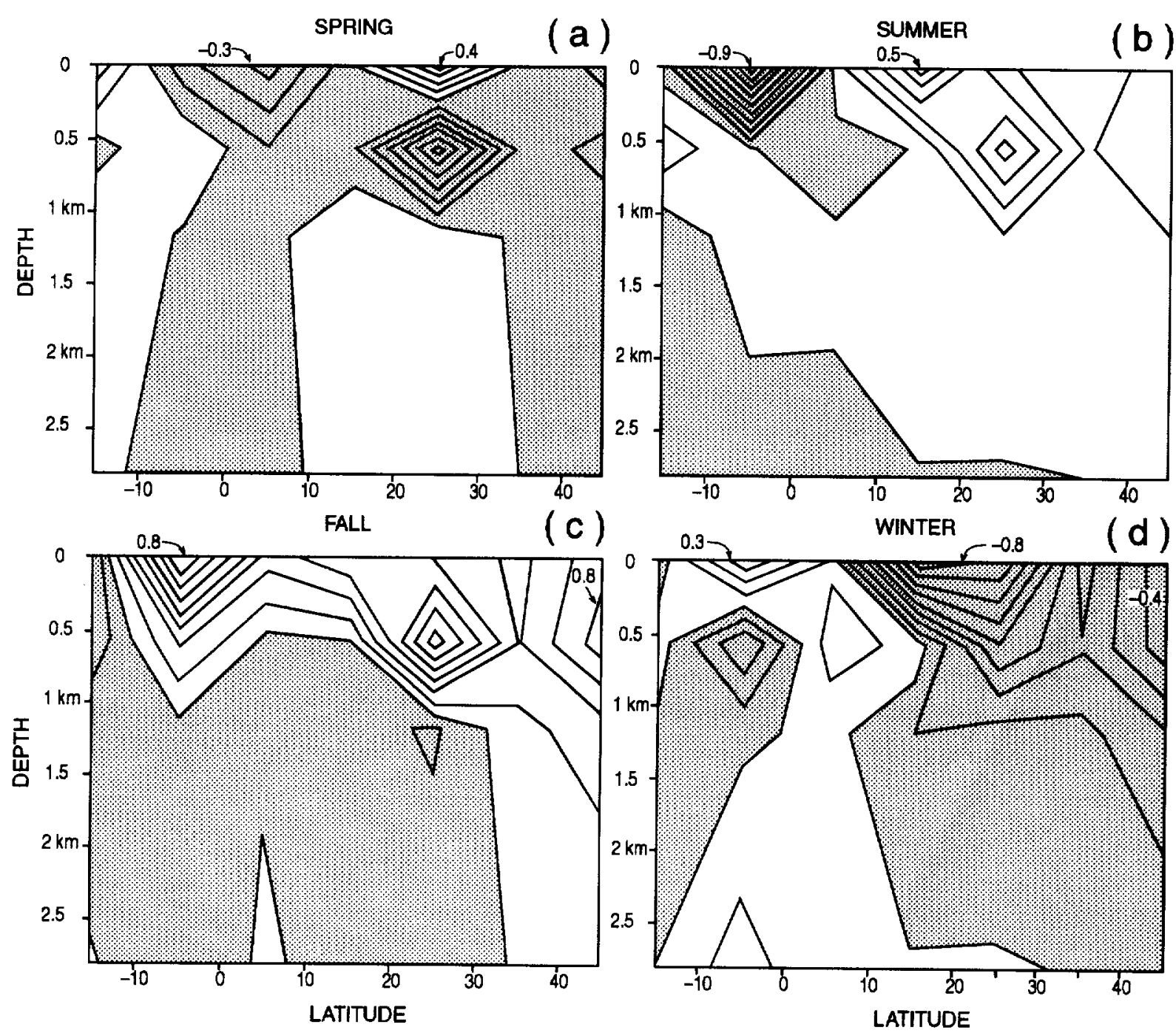

Figure 25. Same as Figure 24, but for estimated seasonal temperature fluctuation. Contour increment is $0.1^{\circ} \mathrm{C}$ in all panels, and negative values are shaded.

residuals can be caused by various processes. The spatial patterns actually seen, however, are strongly suggestive of systematic errors in the $\mathrm{T} / \mathrm{P}$ data, i.e. geoid errors. Stammer and Wunsch [1994] proposed the presence of strong geoid errors in the western tropical $\mathrm{Pa}$ cific, and the inability of the model to reduce the residuals seen in Figure 26 in that area is supporting evidence for the hypothesis. The major competing hypothesis would be that the linear model dynamics are inadequate to reproduce the observations. Although the analysis is not reproduced here, crude estimates suggest that even with this low spatial resolution, the model error is inadequate to explain the residuals. If this conclusion is accepted, the residuals depicted in Figure 26 can be subtracted from the existing geoid estimate to produce an improved geoid. This procedure can be quantified and formalized and involves using the regionally varying estimates of the correlated geoid errors as well as the production of a proper error estimate for the model (see the discussion of Wunsch and Gaposchkin, [1980], on the geoid/circulation estimation problem).

\section{Summary and Discussion}

We have explored the estimation of the ocean circulation from combined surface altimeter observations, a general circulation model, and an associated linear model defined from pressure Green's functions. The Green's function method was applied to two different types of data in the Pacific Ocean. Synthetic data from an identical twin experiment confirmed the potential of the method to estimate the subsurface state of the ocean from the sea surface measurements alone. Reasonable results were also found for state variables which are not directly observed by the altimetry: velocity and temperature fields over the full water column. For observation durations of 1-year or less, there is a loss in resolution in the deepest parts of the water column, a result anticipated by Munk and Wunsch [1982], who proposed that acoustic tomography would be the natural complementary technology. The inference of the need for tomography remains a valid one. What is less clear is whether the deep ocean will become "observable" by 


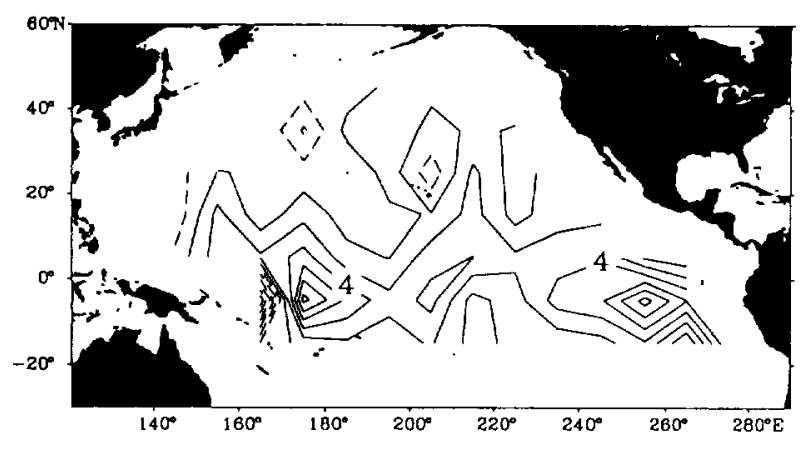

Figure 26. Time mean residuals $\hat{n}=\hat{\delta p}-\delta p_{\text {obs }}$ in equivalent surface elevation obtained at $K=500$ and plotted in geographical order. Contour increment is 2 $\mathrm{cm}$.

altimetry alone if the data span can be extended to decades, when deep ocean structures would have time to manifest themselves at the sea surface.

The main focus here has been on estimating the state of the Pacific Ocean as obtained from 1-year of TOPEX/POSEIDON data, with emphasis on the time average and the annual cycle. Corrections are found to the OGCM initial estimates for these components of the circulation, which are both statistically acceptable relative to prior estimates, and which also pass the test of physical reasonableness. Residuals lying outside the prior statistical uncertainties are strongly geographically correlated and are almost surely a reflection of geoid errors.

At the surface, the resulting time average state reflects a smoothed version of the $\mathrm{T} / \mathrm{P}$ data, with reduced initial dynamically inconsistent anomalies in the data. The correction has weakened the subtropical gyre in the model and confined it mainly to the western portion of the basin. The resulting velocity and temperature fields show horizontal and vertical structures which are consistent with a generally weakened (cooled) subtropical gyre, and the change in mean sea surface is necessarily accompanied by a change in the mean stratification. Despite the shortcomings of the OGCM in computing the annual heating cycle, the seasonal cycle present in the $\mathrm{T} / \mathrm{P}$ observations is successfully imposed on the model, leading to sea surface height changes similar in amplitude and pattern to those observed by $\mathrm{T} / \mathrm{P}$, with a low, cold North Pacific during winter and spring, and a warm, high sea surface during summer and fall. Associated current fluctuations are small, except in the tropical area, where most of the seasonal changes are induced not by local heating, but by changing wind fields. Seasonal fluctuations in surface elevation of the order of a few centimeters are also associated with temperature fluctuations of about $0.5^{\circ}$, which show realistic patterns and amplitudes.

These results are preliminary and can be improved in a number of ways. Use of information on the geographically correlated geoid errors should lead to a significant overall reduction in error. Model spatial resolution and that of the corresponding Green's functions should be greatly increased. Moreover, we have not taken full advantage of all the information available. For example, one should use the twice-daily wind and buoyancy flux fields available from meteorological analyses to improve the OGCM first estimate. Finally, this first attempt was entirely focussed on altimetric data, but there is no reason not to use the same method with all other data types available in the ocean at the same time. In particular, the use of deep constraint information as available from floats and acoustic tomography should produce major improvements in the estimates at depth.

Acknowledgments. Constructive comments by J. Marotzke and P. Jan van Leeuwen are gratefully acknowledged. This study was supported in part by contract 958125 from the Jet Propulsion Laboratory, grant NAGW 1048 from NASA, and NSF grant OCE9205942, and the ATOC experiment of the SERDP program administered by the Advanced Research Projects Agency. Computations were carried out at the National Center for Atmospheric Research, which is sponsored by the National Science Foundation and at the University of Alaska's Arctic Region Supercomputing Center. Contribution to the World Ocean Circulation Experiment.

\section{References}

Benada, R., Merged GDR (TOPEX/POSEIDON) users handbook, JPL Doc. D-11007, Version 1.0, 84 pp., Phys. Oceanogr. Distrib. Active Arch. Cent., Jet Propul. Lab., Pasadena, Calif., 1994.

Bennett, A.F., Inverse Methods in Physical Oceanography, 346 pp., Cambridge University Press, New York, 1992.

Blumen, W., Geostrophic adjustment. Rev. Geophys. ., 10, 485-528, 1972 .

Bolin, B., The adjustment of a non-balanced velocity field towards geostrophic equilibrium in a stratified fluid, Tellus, 5, 373-385, 1953.

Bryan, K., A numerical method for the study of the circulation of the world ocean, J. Comput. Phys., 3, 347-376, 1969.

Cox, M.D., A primitive equation, three-dimensional model of the ocean, GFDL Ocean Group Tech. Rep. 1, Geophys. Fluid Dyn. Lab., Princeton, N.J., 1984.

Fjeldstad, J.E., Ocean currents as an initial problem, Geofys. Publ., Geophys. Norv., 20, 1-24, 1957.

Fukumori, I., J. Benveniste, C. Wunsch, and D.B. Haidvogel, Assimilation of sea surface topography into an ocean circulation model using a steady-state smoother, J. Phys. Oceanogr., 23, 1831-1855, 1993.

Fukumori, I., and P. Malanotte-Rizzoli, An approximate Kalman filter for ocean data assimilation: An example with an idealized Gulf Stream model, J. Geophys. Res., $100,6777-6793,1995$.

Geisler, J.E., Linear theory of the response of a two layer ocean to a moving hurricane, Geophys. Fluid Dyn., 1, 249$272,1970$.

Ghil, M., and P. Malanotte-Rizzoli, Data assimilation in meteorology and oceanography, Adv. in Geophys., 33, 141$266,1991$.

Gill, A.E., Atmosphere-Ocean Dynamics, 662 pp., Academic, San Diego, Calif., 1982.

Gill, A.E., and P.P. Niiler, The theory of the seasonal variability in the ocean, Deep Sea Res., 20, 141-177, 1973. 
Hough, S.S., On the application of harmonic analysis to the dynamical theory of the tides, Part I, On Laplace's oscillations of the first species', and on the dynamics of ocean currents, Philos. Trans. R. Soc. London A, 189, 201-257, 1897.

King, C., D. Stammer, and C. Wunsch, The CMPO/MIT TOPEX/POSEIDON altimetric data set, Rep. 3045 pp., Cent. for Global Change Sci., Mass. Inst. of Technol., Cambridge, Mass., 1994.

Levitus, S., Climatological atlas of the world ocean. NOAA Prof. Pap., 13, 173 pp., 1982.

Longuet-Higgins, M.S., The response of a stratified ocean to stationary or moving wind systems, Deep Sea Res., 12, 923-973, 1965.

Ma, X.C., C.K. Shum, R.J. Eanes, and B.D. Tapley, Determination of ocean tides from the first year of TOPEX/POSEIDON altimeter measurements, J. Geophys. Res., 99, 24,809-24,820, 1994.

Marotzke, J., and C. Wunsch, Finding the steady state of a general circulation model through data assimilation: Application to the North Atlantic Ocean, J. Geophys. Res., 98, 20,149-20,167, 1993.

Memery, L., and C. Wunsch, Constraining the North Atlantic Circulation with tritium data, J. Geophys. Res., 95, 5239-5256, 1990.

Menemenlis, D., and C. Wunsch, Linearization of an oceanic general circulation model for data assimilation and climate studies, J. Atmos. Oceanic. Technol., in press, 1996.

Morse, P.M., and H. Feshbach, Methods of Theoretical Physics, Part 1, 997 pp., McGraw-Hill Book, New York, 1953.

Munk, W. and C. Wunsch, Observing the ocean in the 1990s, Philos. Trans. R. Soc. London A, 307, 439-464, 1982.

Nerem, R. S. et al., Gravity model development for TOPEX/POSEIDON: Joint gravity model 1 and $2, J$. Geophys. Res., 99, 24,405-24,448, 1994.

Philander, S.G.H., Forced oceanic waves, Rev. Geophys., 16, 15-46, 1978.

Ponte, R. M., The sea level response of a stratified ocean to barometric pressure forcing. J. Phys. Oceanogr., 22, 109-113, 1992.

Rapp, R.H., Y.M. Ming, and N.K. Pavlis, The Ohio State 1991 Geopotential and Sea Surface Topography Harmonic Coefficient Model, Rep. 410, Dep. of Geod. Sci. and Surv., Ohio State Univ., Columbus, 1991.

Roach, G.F., Green's Functions, 325 pp., Cambridge Univ. Press, New York, 1982.

Rossby, C.G., Dynamics of steady ocean currents in the light of experimental fluid mechanics, Pap. Phys. Oceanogr. Meteorol., 5 (1), 43 pp., 1936.
Sarmiento, J. L., On the North and tropical Atlantic heat balance, J. Geophys. Res., 91, 11,677-11,689, 1986.

Sarmiento, J. L., and K. Bryan, An ocean transport model for the North Atlantic, J. Geophys. Res., 87, 394-408, 1982.

Semtner, A.J., and R.M. Chervin, Ocean general circulation from a global eddy-resolving model, J. Geophys. Res., 97, 5493-5550, 1992.

Stammer, D. and C. Wunsch, Preliminary assessment of the accuracy and precision of TOPEX/POSEIDON altimeter data with respect to the large scale ocean circulation. $J$. Geophys. Res., 99, 24,584-25,604, 1994.

Stammer, D., R. Tokmakian, A. Semtner, and C. Wunsch, How well does a $1 / 4^{\circ}$ global circulation model simulate large-scale oceanic observations? J. Geophys. Res., in press, 1996.

Trenberth, K.E., J.G. Olson, and W.G. Large, A global ocean wind stress climatology based ECMWF analyses, NCAR Tech. Note, TN-338+STR, , 93 pp., Natl. Cent. for Atmos. Res., Boulder, Colo., 1989.

Tsaoussi, L.S., and C.J. Koblinsky, An error covariance model for the sea surface topography and velocity derived from TOPEX/POSEIDON altimetry, J. Geophys. Res., $99,24,669-24,684,1994$.

Van Huffel, S., and J. Vandewalle, The Total Least Squares Problem, Computational A spects and Analysis, SIAM, 300 pp., Philadelphia, Pa., 1991.

Wunsch, C., Eclectic modeling of the North Atlantic (II), Transient tracers and the ventilation of the eastern basin thermocline, Philos. Trans. R. Soc. London, A, 325, 201236,1988 .

Wunsch, C., The Ocean Circulation Inverse Problem, 442 pp., Cambridge Univ. Press, New York, 1996.

Wunsch, C., and E.M. Gaposchkin, On using satellite altimetry to determine the general circulation of the ocean with application to geoid improvements, Rev. Geophys., $18,725-745,1980$.

Wunsch, C., and D. Stammer, Atmospheric loading and the "inverted barometer" effect, Rev. Geophys., in press, 1996.

D. Stammer and C. Wunsch, Center for Meteorology and Physical Oceanography, Department of Earth, Atmospheric and Planetary Sciences, Massachusetts Institute of Technology, Cambridge, MA 02139. (e-mail: detlef@lagoon.mit.edu; cwunsch@pond.mit.edu)

(Received April 7, 1995; revised April 2, 1996; accepted April 18, 1996.) 